Network Working Group

Request for Comments: 2910

Obsoletes: 2565

Category: Standards Track
R. Herriot, Editor

Xerox Corporation

S. Butler

Hewlett-Packard

P. Moore

Peerless Systems Networking

R. Turner

2wire.com

J. Wenn

Xerox Corporation

September 2000

Internet Printing Protocol/1.1: Encoding and Transport

Status of this Memo

This document specifies an Internet standards track protocol for the Internet community, and requests discussion and suggestions for improvements. Please refer to the current edition of the "Internet Official Protocol Standards" (STD 1) for the standardization state and status of this protocol. Distribution of this memo is unlimited.

Copyright Notice

Copyright (C) The Internet Society (2000). All Rights Reserved.

Abstract

This document is one of a set of documents, which together describe all aspects of a new Internet Printing Protocol (IPP). IPP is an application level protocol that can be used for distributed printing using Internet tools and technologies. This document defines the rules for encoding IPP operations and IPP attributes into a new Internet mime media type called "application/ipp". This document also defines the rules for transporting over Hypertext Transfer Protocol (HTTP) a message body whose Content-Type is "application/ipp". This document defines a new scheme named 'ipp' for identifying IPP printers and jobs. 
The full set of IPP documents includes:

Design Goals for an Internet Printing Protocol [RFC2567]

Rationale for the Structure and Model and Protocol for the Internet Printing Protocol [RFC2568]

Internet Printing Protocol/1.1: Model and Semantics [RFC2911]

Internet Printing Protocol/1.1: Encoding and Transport (this document)

Internet Printing Protocol/1.1: Implementer's Guide [ipp-iig]

Mapping between LPD and IPP Protocols [RFC2569]

The document, "Design Goals for an Internet Printing Protocol", takes a broad look at distributed printing functionality, and it enumerates real-life scenarios that help to clarify the features that need to be included in a printing protocol for the Internet. It identifies requirements for three types of users: end users, operators, and administrators. It calls out a subset of end user requirements that are satisfied in IPP/1.1. A few OPTIONAL operator operations have been added to IPP/1.1.

The document, "Rationale for the Structure and Model and Protocol for the Internet Printing Protocol", describes IPP from a high level view, defines a roadmap for the various documents that form the suite of IPP specification documents, and gives background and rationale for the IETF working group's major decisions.

The document, "Internet Printing Protocol/1.1: Model and Semantics", describes a simplified model with abstract objects, their attributes, and their operations that are independent of encoding and transport. It introduces a Printer and a Job object. The Job object optionally supports multiple documents per Job. It also addresses security, internationalization, and directory issues.

The document "Internet Printing Protocol/1.1: Implementer's Guide", gives advice to implementers of IPP clients and IPP objects.

The document "Mapping between LPD and IPP Protocols", gives some advice to implementers of gateways between IPP and LPD (Line Printer Daemon) implementations. 
Table of Contents

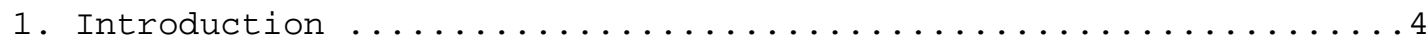

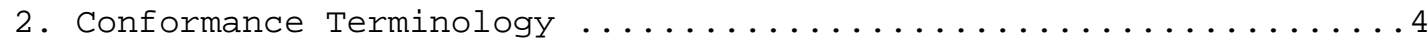

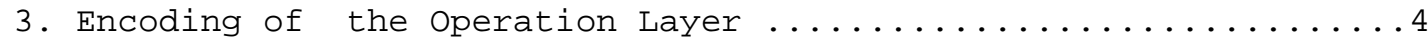

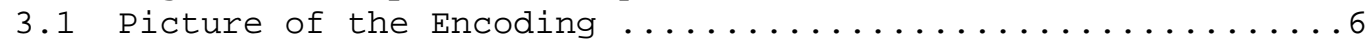

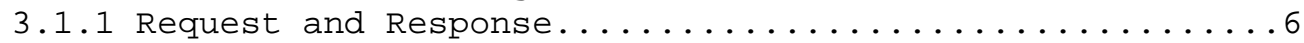

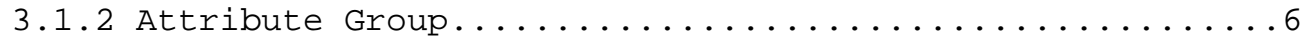

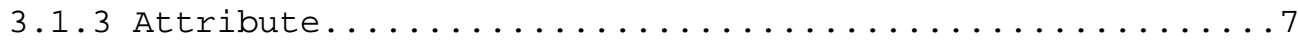

3.1.4 Picture of the Encoding of an Attribute-with-one-value.7

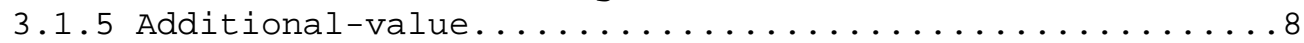

3.1.6 Alternative Picture of the Encoding of a Request or a

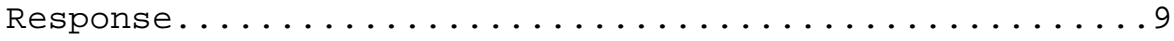

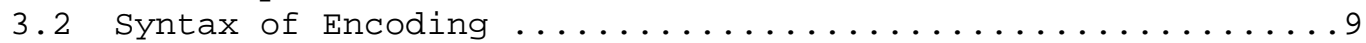

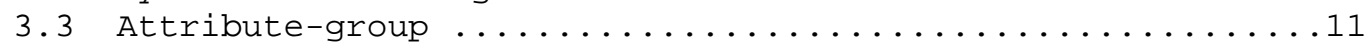

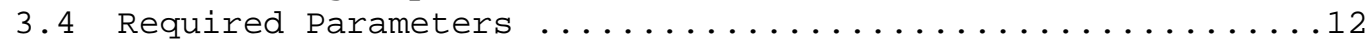

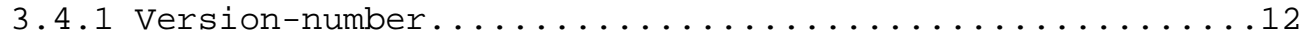

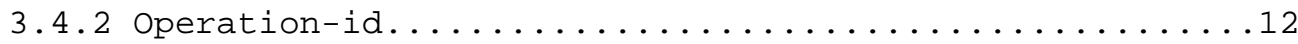

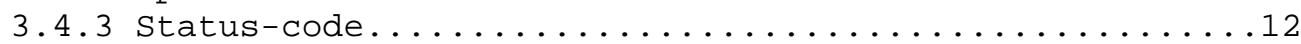

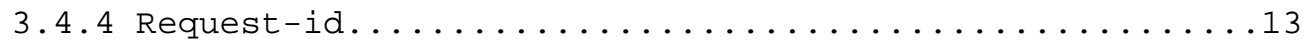

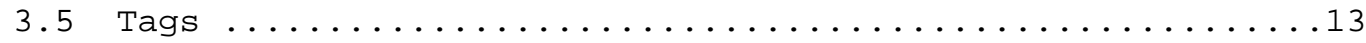

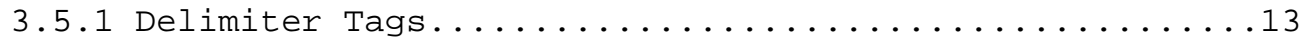

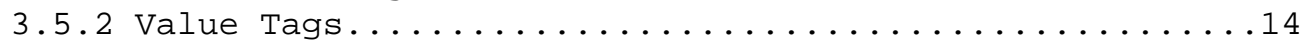

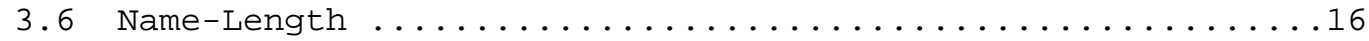

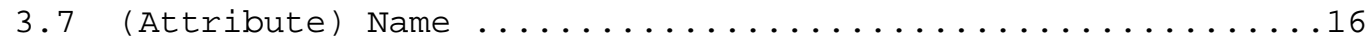

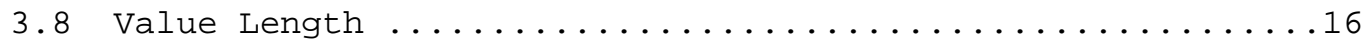

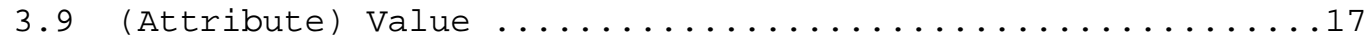

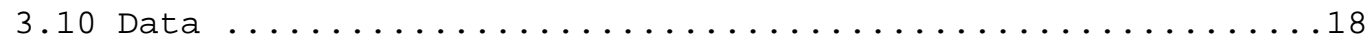

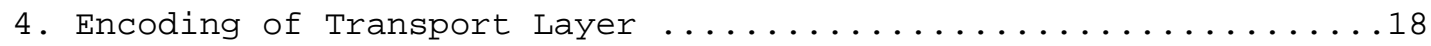

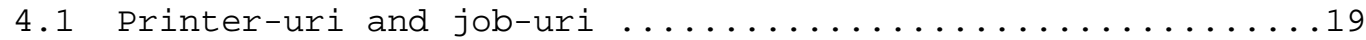

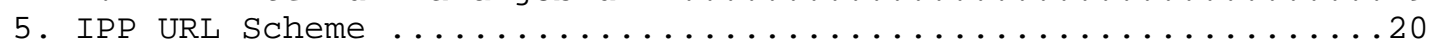

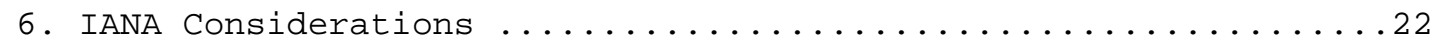

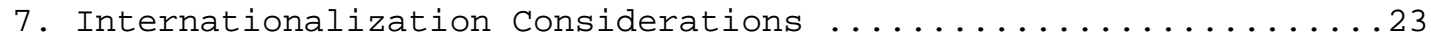

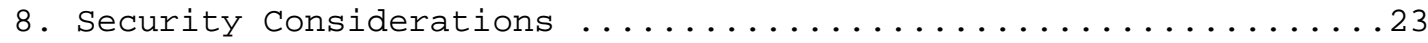

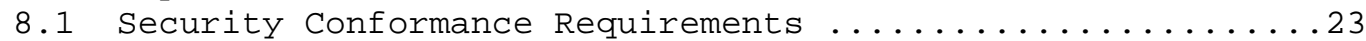

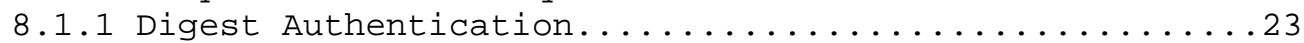

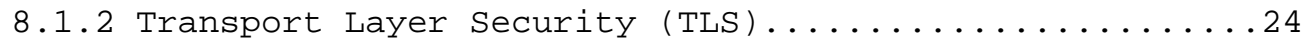

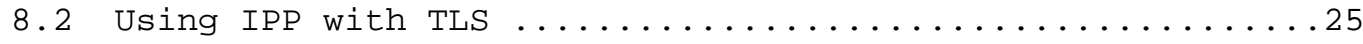

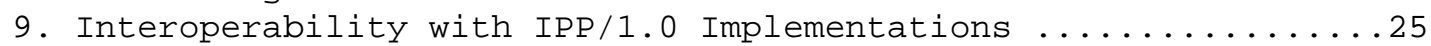

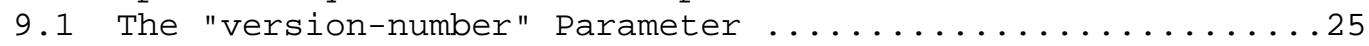

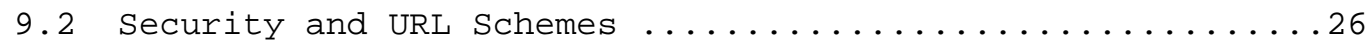

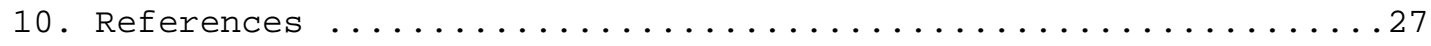

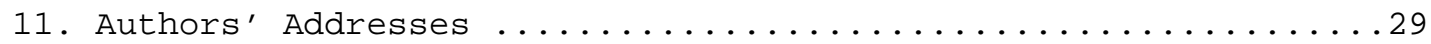

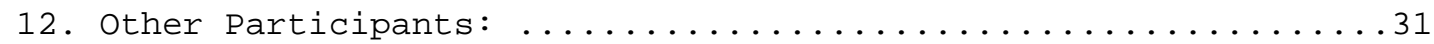

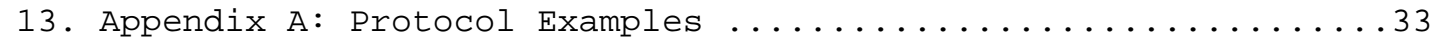

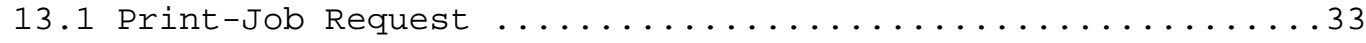

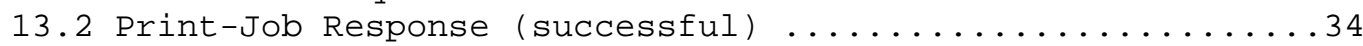

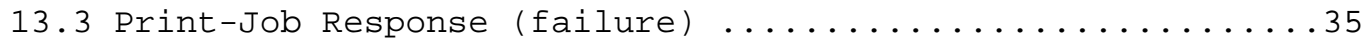


13.4 Print-Job Response (success with attributes ignored) ....36

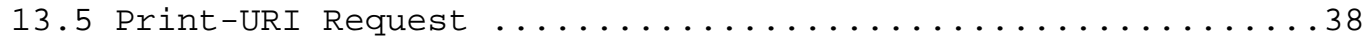

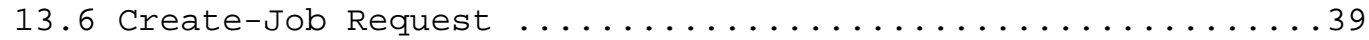

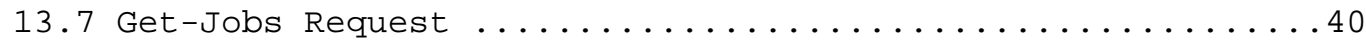

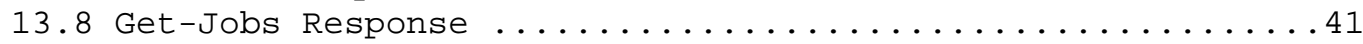

14. Appendix B: Registration of MIME Media Type Information for

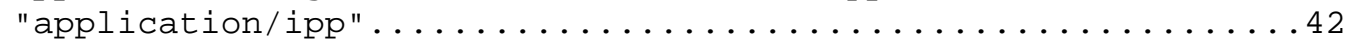

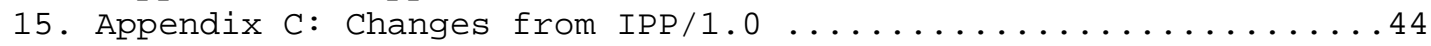

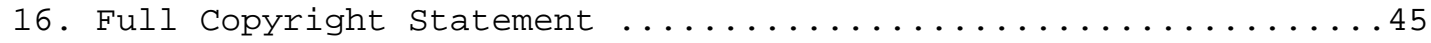

1. Introduction

This document contains the rules for encoding IPP operations and describes two layers: the transport layer and the operation layer.

The transport layer consists of an HTTP/1.1 request or response. RFC 2616 [RFC2616] describes HTTP/1.1. This document specifies the HTTP headers that an IPP implementation supports.

The operation layer consists of a message body in an HTTP request or response. The document "Internet Printing Protocol/1.1: Model and Semantics" [RFC2911] defines the semantics of such a message body and the supported values. This document specifies the encoding of an IPP operation. The aforementioned document [RFC2911] is henceforth referred to as the "IPP model document" or simply "model document".

Note: the version number of IPP (1.1) and HTTP (1.1) are not linked. They both just happen to be 1.1 .

\section{Conformance Terminology}

The key words "MUST", "MUST NOT", "REQUIRED", "SHOULD", "SHOULD NOT", "RECOMMENDED", "MAY", and "OPTIONAL" in this document are to be interpreted as described in RFC 2119 [RFC2119].

3. Encoding of the Operation Layer

The operation layer is the message body part of the HTTP request or response and it MUST contain a single IPP operation request or IPP operation response. Each request or response consists of a sequence of values and attribute groups. Attribute groups consist of a sequence of attributes each of which is a name and value. Names and values are ultimately sequences of octets.

The encoding consists of octets as the most primitive type. There are several types built from octets, but three important types are integers, character strings and octet strings, on which most other data types are built. Every character string in this encoding MUST be 
a sequence of characters where the characters are associated with some charset and some natural language. A character string MUST be in "reading order" with the first character in the value (according to reading order) being the first character in the encoding. A character string whose associated charset is US-ASCII whose associated natural language is US English is henceforth called a US-ASCII-STRING. A character string whose associated charset and natural language are specified in a request or response as described in the model document is henceforth called a LOCALIZED-STRING. An octet string MUST be in "IPP model document order" with the first octet in the value (according to the IPP model document order) being the first octet in the encoding. Every integer in this encoding MUST be encoded as a signed integer using two's-complement binary encoding with big-endian format (also known as "network order" and "most significant byte first"). The number of octets for an integer MUST be 1, 2 or 4, depending on usage in the protocol. Such one-octet integers, henceforth called SIGNED-BYTE, are used for the version-number and tag fields. Such two-byte integers, henceforth called SIGNED-SHORT are used for the operation-id, status-code and length fields. Four byte integers, henceforth called SIGNED-INTEGER, are used for value fields and the request-id.

The following two sections present the encoding of the operation layer in two ways:

- informally through pictures and description

- formally through Augmented Backus-Naur Form (ABNF), as specified by RFC 2234 [RFC2234]

An operation request or response MUST use the encoding described in these two sections. 


\subsection{Picture of the Encoding}

3.1.1 Request and Response

An operation request or response is encoded as follows:

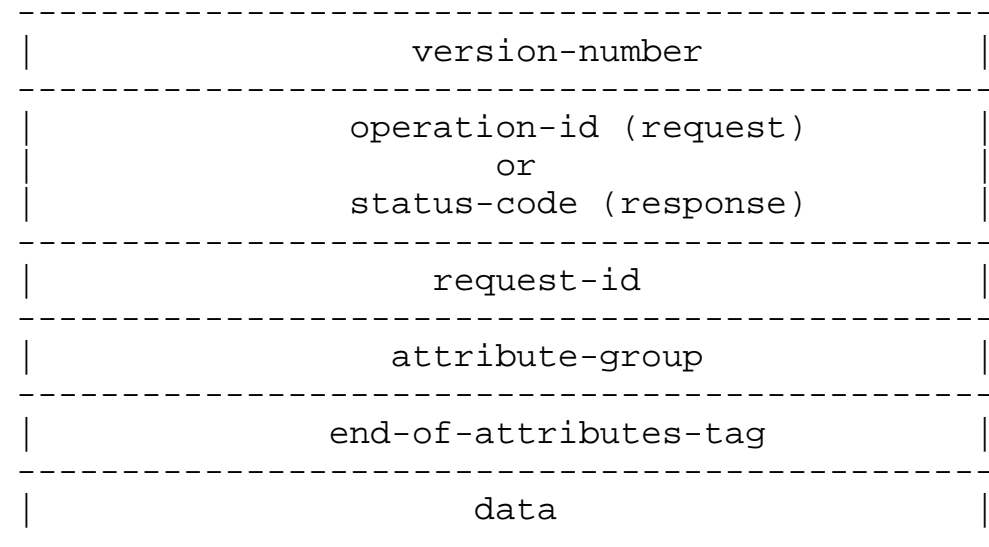

2 bytes - required

2 bytes - required

4 bytes - required

$\mathrm{n}$ bytes - 0 or more

1 byte - required

q bytes - optional

The first three fields in the above diagram contain the value of attributes described in section 3.1 .1 of the Model document.

The fourth field is the "attribute-group" field, and it occurs o or more times. Each "attribute-group" field represents a single group of attributes, such as an Operation Attributes group or a Job Attributes group (see the Model document). The IPP model document specifies the required attribute groups and their order for each operation request and response.

The "end-of-attributes-tag" field is always present, even when the "data" is not present. The Model document specifies for each operation request and response whether the "data" field is present or absent.

\subsubsection{Attribute Group}

Each "attribute-group" field is encoded as follows:

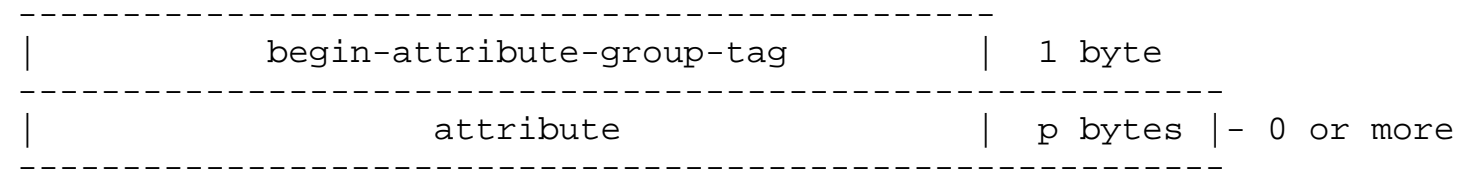


The "begin-attribute-group-tag" field marks the beginning of an "attribute-group" field and its value identifies the type of attribute group, e.g. Operations Attributes group versus a Job Attributes group. The "begin-attribute-group-tag" field also marks the end of the previous attribute group except for the "beginattribute-group-tag" field in the first "attribute-group" field of a request or response. The "begin-attribute-group-tag" field acts as an "attribute-group" terminator because an "attribute-group" field cannot nest inside another "attribute-group" field.

An "attribute-group" field contains zero or more "attribute" fields.

Note, the values of the "begin-attribute-group-tag" field and the "end-of-attributes-tag" field are called "delimiter-tags".

\subsubsection{Attribute}

An "attribute" field is encoded as follows:

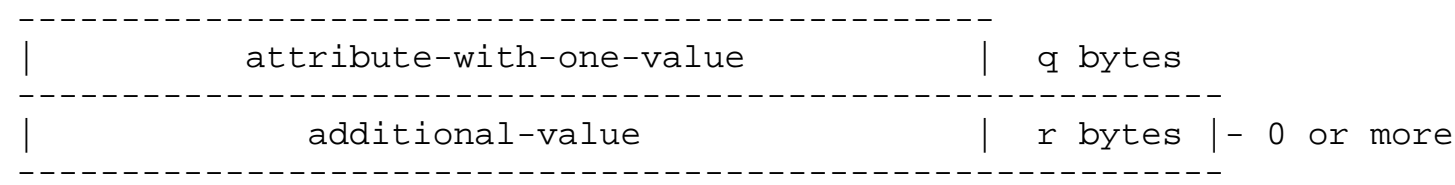

When an attribute is single valued (e.g. "copies" with value of 10) or multi-valued with one value (e.g. "sides-supported" with just the value 'one-sided') it is encoded with just an "attribute-with-onevalue" field. When an attribute is multi-valued with $n$ values (e.g. "sides-supported" with the values 'one-sided' and 'two-sided-longedge'), it is encoded with an "attribute-with-one-value" field followed by n-1 "additional-value" fields.

3.1.4 Picture of the Encoding of an Attribute-with-one-value Each "attribute-with-one-value" field is encoded as follows:

\begin{tabular}{|c|c|}
\hline | & value-tag \\
\hline | & name-length (value is u) \\
\hline $\mid$ & name \\
\hline | & value-length (value is v) \\
\hline $\mid$ & value \\
\hline
\end{tabular}

1 byte

2 bytes

u bytes

2 bytes

$\mathrm{v}$ bytes 
An "attribute-with-one-value" field is encoded with five subfields:

The "value-tag" field specifies the attribute syntax, e.g. 0x44

for the attribute syntax 'keyword'.

The "name-length" field specifies the length of the "name" field in bytes, e.g. u in the above diagram or 15 for the name "sidessupported".

The "name" field contains the textual name of the attribute, e.g. "sides-supported".

The "value-length" field specifies the length of the "value" field in bytes, e.g. $V$ in the above diagram or 9 for the (keyword) value ' one-sided'.

The "value" field contains the value of the attribute, e.g. the textual value 'one-sided'.

3.1.5 Additional-value

Each "additional-value" field is encoded as follows:

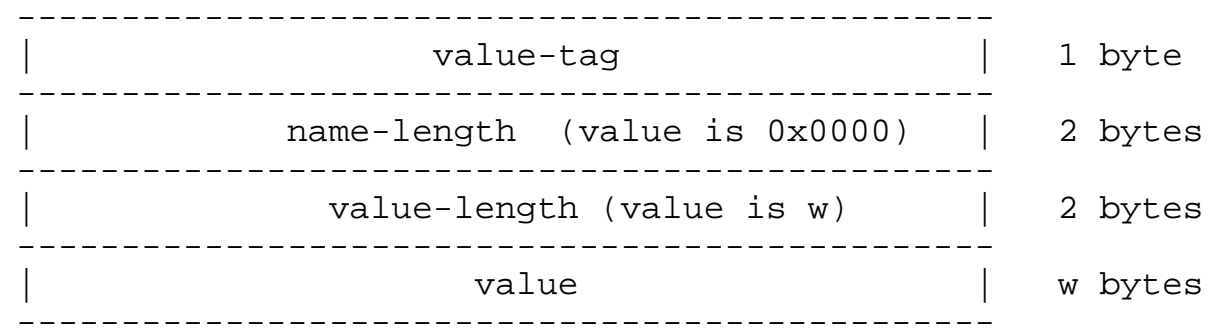

An "additional-value" is encoded with four subfields:

The "value-tag" field specifies the attribute syntax, e.g. 0x44 for the attribute syntax 'keyword'.

The "name-length" field has the value of 0 in order to signify that it is an "additional-value". The value of the "name-length" field distinguishes an "additional-value" field ("name-length" is 0) from an "attribute-with-one-value" field ("name-length" is not $0)$.

The "value-length" field specifies the length of the "value" field in bytes, e.g. w in the above diagram or 19 for the (keyword) value 'two-sided-long-edge'. 
The "value" field contains the value of the attribute, e.g. the textual value 'two-sided-long-edge'.

3.1.6 Alternative Picture of the Encoding of a Request Or a Response From the standpoint of a parser that performs an action based on a "tag" value, the encoding consists of:

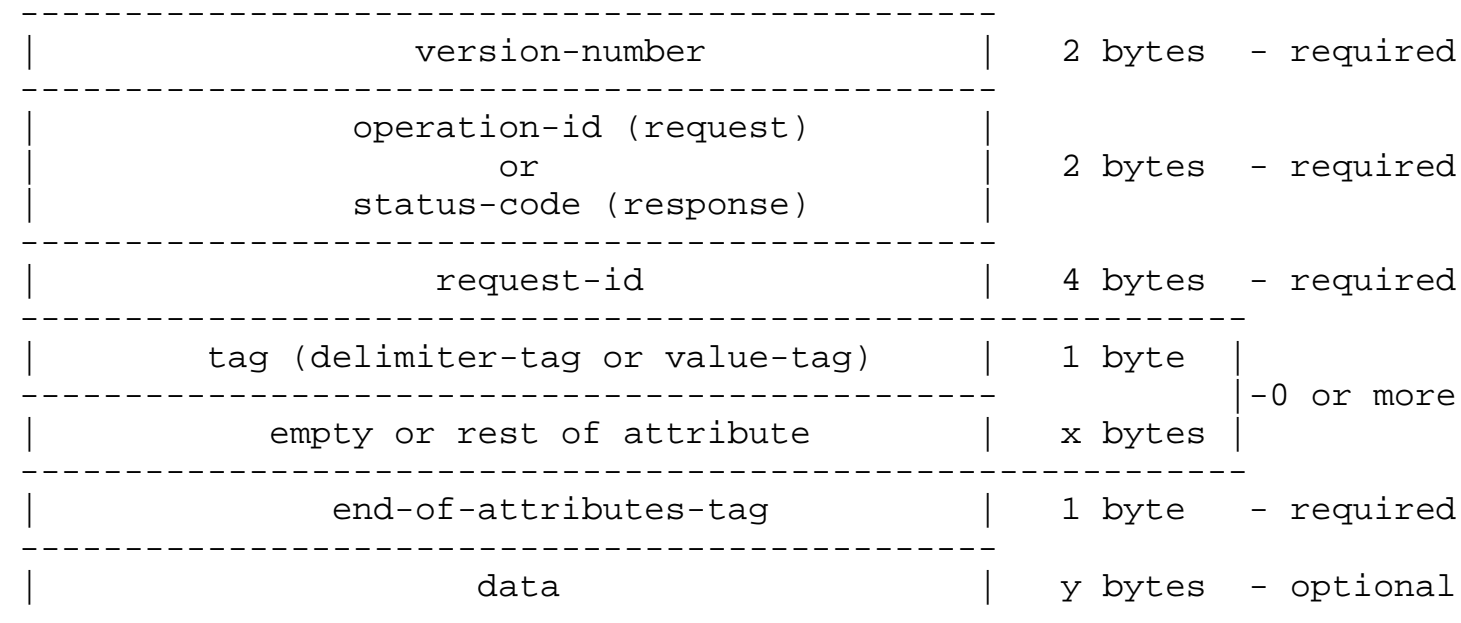

The following show what fields the parser would expect after each type of "tag":

- "begin-attribute-group-tag": expect zero or more "attribute" fields

- "value-tag": expect the remainder of an "attribute-with-onevalue" or an "additional-value".

- "end-of-attributes-tag": expect that "attribute" fields are complete and there is optional "data"

\subsection{Syntax of Encoding}

The syntax below is ABNF [RFC2234] except 'strings of literals' MUST be case sensitive. For example ' $a$ ' means lower case ' $a$ ' and not upper case ' $A$ '. In addition, SIGNED-BYTE and SIGNED-SHORT fields are represented as ' $\% x^{\prime}$ values which show their range of values.

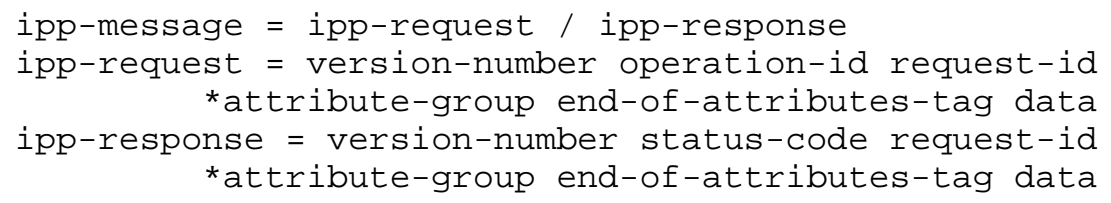




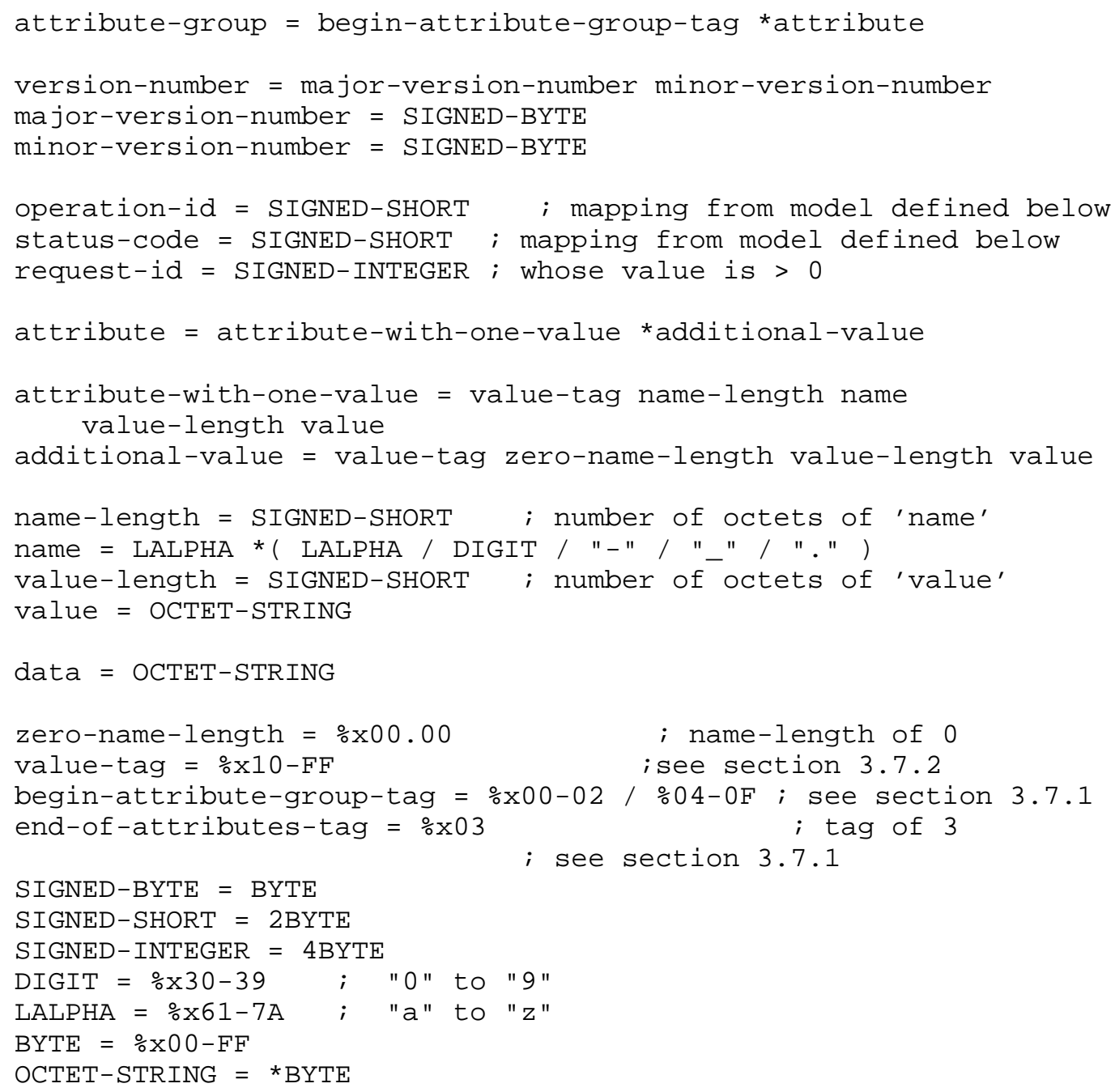

The syntax below defines additional terms that are referenced in this document. This syntax provides an alternate grouping of the delimiter tags.

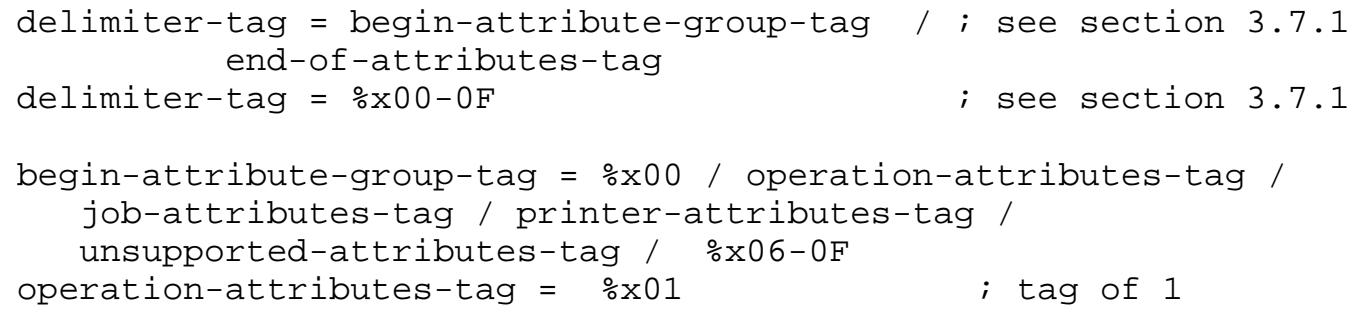




$\begin{array}{ll}\text { job-attributes-tag }=\circ \times 02 & \text {; tag of } 2 \\ \text { printer-attributes-tag }=\circ \times 04 & \text {; tag of } 4 \\ \text { unsupported-attributes-tag }=\circ \times 05 & \text {; tag of } 5\end{array}$

\subsection{Attribute-group}

Each "attribute-group" field MUST be encoded with the "beginattribute-group-tag" field followed by zero or more "attribute" subfields.

The table below maps the model document group name to value of the "begin-attribute-group-tag" field:

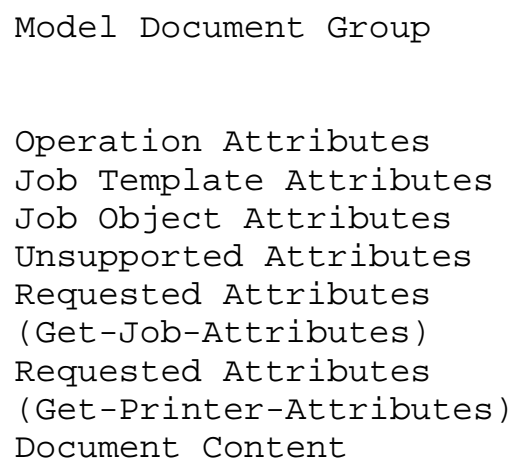

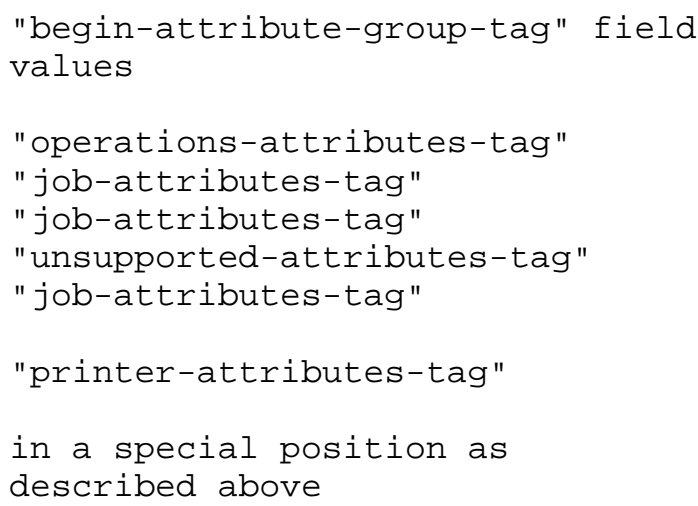

For each operation request and response, the model document prescribes the required and optional attribute groups, along with their order. Within each attribute group, the model document prescribes the required and optional attributes, along with their order.

When the Model document requires an attribute group in a request or response and the attribute group contains zero attributes, a request or response SHOULD encode the attribute group with the "beginattribute-group-tag" field followed by zero "attribute" fields. For example, if the client requests a single unsupported attribute with the Get-Printer-Attributes operation, the Printer MUST return no "attribute" fields, and it SHOULD return a "begin-attribute-grouptag" field for the Printer Attributes Group. The Unsupported Attributes group is not such an example. According to the model document, the Unsupported Attributes Group SHOULD be present only if the unsupported attributes group contains at least one attribute.

A receiver of a request MUST be able to process the following as equivalent empty attribute groups: 
a) A "begin-attribute-group-tag" field with zero following "attribute" fields.

b) An expected but missing "begin-attribute-group-tag" field.

When the Model document requires a sequence of an unknown number of attribute groups, each of the same type, the encoding MUST contain one "begin-attribute-group-tag" field for each attribute group even when an "attribute-group" field contains zero "attribute" sub-fields. For example, for the Get-Jobs operation may return zero attributes for some jobs and not others. The "begin-attribute-group-tag" field followed by zero "attribute" fields tells the recipient that there is a job in queue for which no information is available except that it is in the queue.

\subsection{Required Parameters}

Some operation elements are called parameters in the model document [RFC2911]. They MUST be encoded in a special position and they MUST NOT appear as operation attributes. These parameters are described in the subsections below.

\subsubsection{Version-number}

The "version-number" field MUST consist of a major and minor version-number, each of which MUST be represented by a SIGNED-BYTE. The major version-number MUST be the first byte of the encoding and the minor version-number MUST be the second byte of the encoding. The protocol described in this document MUST have a major version-number of 1 (0x01) and a minor version-number of 1 (0x01). The ABNF for these two bytes MUST be x01.01.

\section{4 .2 Operation-id}

The "operation-id" field MUST contain an operation-id value defined in the model document. The value MUST be encoded as a SIGNED-SHORT and it MUST be in the third and fourth bytes of the encoding of an operation request.

\subsection{3 status-code}

The "status-code" field MUST contain a status-code value defined in the model document. The value MUST be encoded as a SIGNED-SHORT and it MUST be in the third and fourth bytes of the encoding of an operation response. 
The status-code is an operation attribute in the model document. In the protocol, the status-code is in a special position, outside of the operation attributes.

If an IPP status-code is returned, then the HTTP Status-Code MUST be 200 (successful-ok). With any other HTTP Status-Code value, the HTTP response MUST NOT contain an IPP message-body, and thus no IPP status-code is returned.

\subsubsection{Request-id}

The "request-id" field MUST contain a request-id value as defined in the model document. The value MUST be encoded as a SIGNED-INTEGER and it MUST be in the fifth through eighth bytes of the encoding.

\subsection{Tags}

There are two kinds of tags:

- delimiter tags: delimit major sections of the protocol, namely attributes and data

- value tags: specify the type of each attribute value

3.5.1 Delimiter Tags

The following table specifies the values for the delimiter tags:

Tag Value (Hex) Meaning

0x00 reserved for definition in a future IETF standards track document

0x01 "operation-attributes-tag"

0x02 "job-attributes-tag"

0x03 "end-of-attributes-tag"

0x04 "printer-attributes-tag"

0x05 "unsupported-attributes-tag"

0x06-0x0f reserved for future delimiters in IETF standards track documents

When a "begin-attribute-group-tag" field occurs in the protocol, it means that zero or more following attributes up to the next delimiter tag MUST be attributes belonging to the attribute group specified by the value of the "begin-attribute-group-tag". For example, if the value of "begin-attribute-group-tag" is 0x01, the following attributes MUST be members of the Operations Attributes group. 
The "end-of-attributes-tag" (value 0x03) MUST occur exactly once in an operation. It MUST be the last "delimiter-tag". If the operation has a document-content group, the document data in that group MUST follow the "end-of-attributes-tag".

The order and presence of "attribute-group" fields (whose beginning is marked by the "begin-attribute-group-tag" subfield) for each operation request and each operation response MUST be that defined in the model document. For further details, see section 3.7 " (Attribute) Name" and 13 "Appendix A: Protocol Examples".

A Printer MUST treat a "delimiter-tag" (values from 0x00 through 0x0F) differently from a "value-tag" (values from 0x10 through 0xFF) so that the Printer knows that there is an entire attribute group that it doesn't understand as opposed to a single value that it doesn't understand.

\subsubsection{Value Tags}

The remaining tables show values for the "value-tag" field, which is the first octet of an attribute. The "value-tag" field specifies the type of the value of the attribute.

The following table specifies the "out-of-band" values for the "value-tag" field.

Tag Value (Hex) Meaning

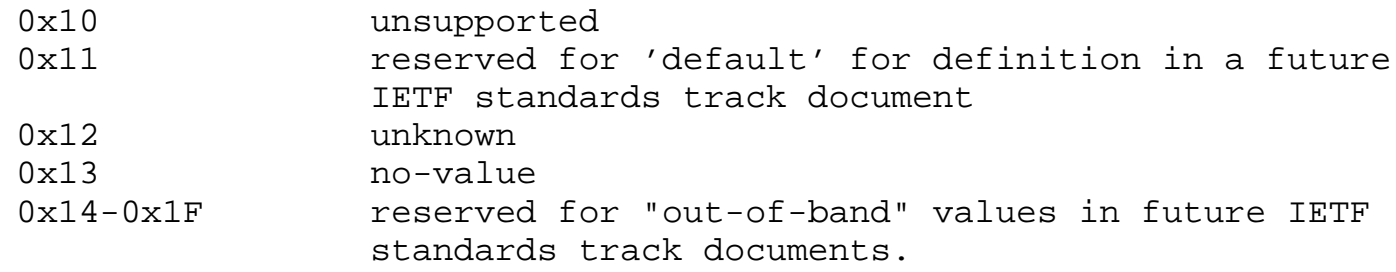


NOTE: 0x20 is reserved for "generic integer" if it should ever be needed.

The following table specifies the octetstring values for the "valuetag" field:

Tag Value (Hex) Meaning

$0 \times 30$ octetstring with an unspecified format

$0 \times 31$ dateTime

$0 \times 32 \quad$ resolution

$0 \times 33 \quad$ rangeofinteger

0x34 reserved for definition in a future IETF

$0 \times 35 \quad$ textWithLanguage

$0 \times 36 \quad$ nameWithLanguage

0x37-0x3F reserved for octetstring type definitions in

future IETF standards track documents

The following table specifies the character-string values for the "value-tag" field:

Tag Value (Hex) Meaning

0x40 reserved for definition in a future IETF

standards track document

$0 \times 41$ textwithoutLanguage

$0 \times 42 \quad$ nameWithoutLanguage

0x43 reserved for definition in a future IETF

$0 \times 44 \quad$ keyword

$0 \times 45 \quad$ uri

0x46 urischeme

$0 \times 47 \quad$ charset

$0 \times 48 \quad$ naturallanguage

0x49 mimeMediaType

0x4A-0x5F reserved for character string type definitions in future IETF standards track documents

NOTE: $0 \times 40$ is reserved for "generic character-string" if it should ever be needed.

NOTE: an attribute value always has a type, which is explicitly specified by its tag; one such tag value is "nameWithoutlanguage". An attribute's name has an implicit type, which is keyword.

The values $0 \times 60-0 \times F F$ are reserved for future type definitions in IETF standards track documents. 
The tag $0 \times 7 F$ is reserved for extending types beyond the 255 values available with a single byte. A tag value of 0x7F MUST signify that the first 4 bytes of the value field are interpreted as the tag value. Note this future extension doesn't affect parsers that are unaware of this special tag. The tag is like any other unknown tag, and the value length specifies the length of a value, which contains a value that the parser treats atomically. Values from 0x00 to 0x37777777 are reserved for definition in future IETF standard track documents. The values 0x40000000 to 0x7FFFFFFF are reserved for vendor extensions.

\section{6 Name-Length}

The "name-length" field MUST consist of a SIGNED-SHORT. This field MUST specify the number of octets in the immediately following "name" field. The value of this field excludes the two bytes of the "namelength" field. For example, if the "name" field contains "sides", the value of this field is 5 .

If a "name-length" field has a value of zero, the following "name" field MUST be empty, and the following value MUST be treated as an additional value for the attribute encoded in the nearest preceding "attribute-with-one-value" field. Within an attribute group, if two or more attributes have the same name, the attribute group is malformed (see [RFC2911] section 3.1.3). The zero-length name is the only mechanism for multi-valued attributes.

\section{7 (Attribute) Name}

The "name" field MUST contain the name of an attribute. The model document [RFC2911] specifies such names.

\subsection{Value Length}

The "value-length" field MUST consist of a SIGNED-SHORT. This field MUST specify the number of octets in the immediately following "value" field. The value of this field excludes the two bytes of the "value-length" field. For example, if the "value" field contains the keyword (text) value 'one-sided', the value of this field is 9.

For any of the types represented by binary signed integers, the sender MUST encode the value in exactly four octets.

For any of the types represented by character-strings, the sender MUST encode the value with all the characters of the string and without any padding characters. 


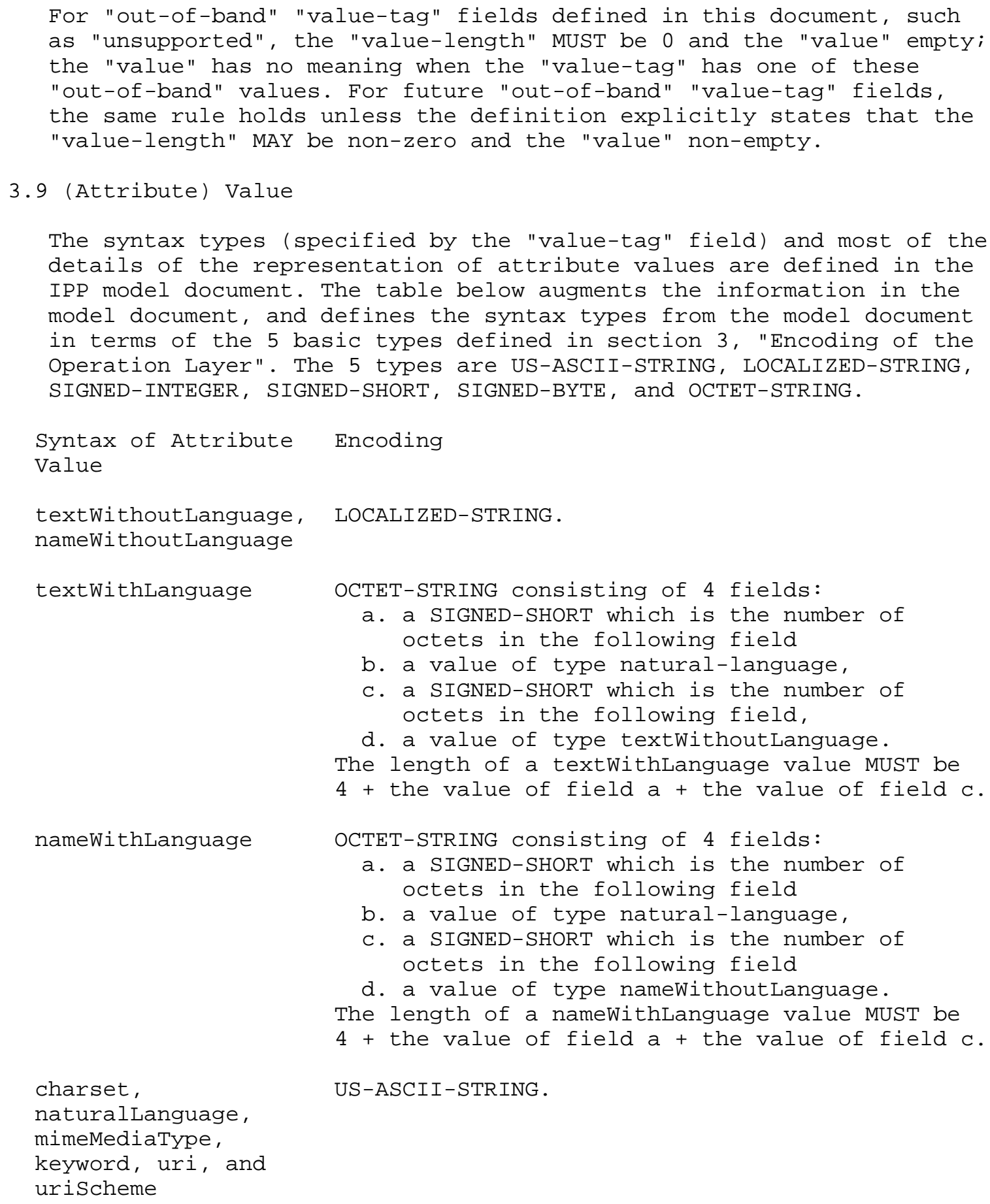




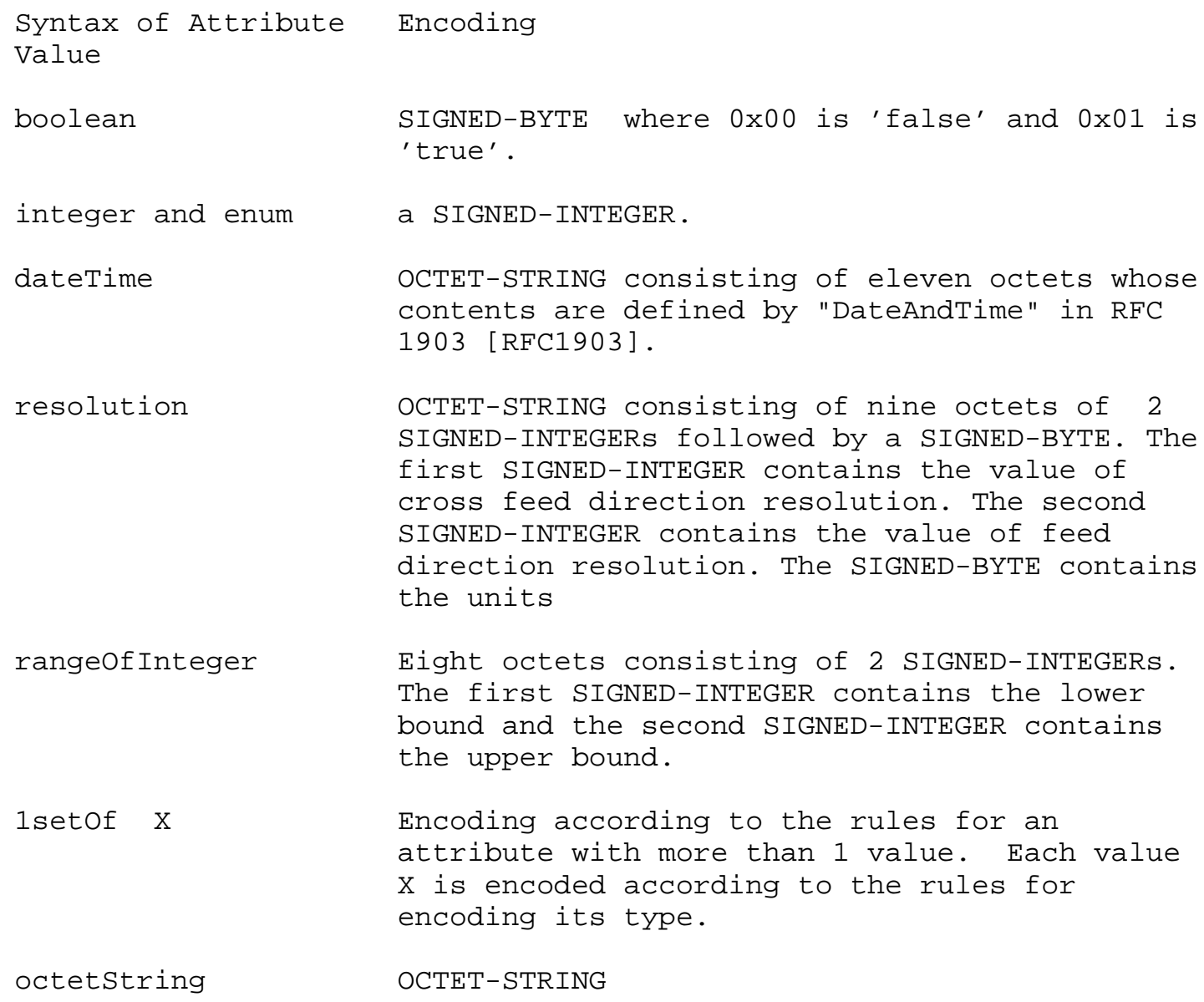

The attribute syntax type of the value determines its encoding and the value of its "value-tag".

3.10 Data

The "data" field MUST include any data required by the operation

4. Encoding of Transport Layer

HTTP/1.1 [RFC2616] is the transport layer for this protocol.

The operation layer has been designed with the assumption that the transport layer contains the following information: 
- the URI of the target job or printer operation

- the total length of the data in the operation layer, either as a single length or as a sequence of chunks each with a length.

It is REQUIRED that a printer implementation support HTTP over the IANA assigned Well known Port 631 (the IPP default port), though a printer implementation may support HTTP over some other port as well.

Each HTTP operation MUST use the POST method where the request-URI is the object target of the operation, and where the "Content-Type" of the message-body in each request and response MUST be "application/ipp". The message-body MUST contain the operation layer and MUST have the syntax described in section 3.2 "Syntax of Encoding". A client implementation MUST adhere to the rules for a client described for HTTP1.1 [RFC2616]. A printer (server) implementation MUST adhere the rules for an origin server described for HTTP1.1 [RFC2616].

An IPP server sends a response for each request that it receives. If an IPP server detects an error, it MAY send a response before it has read the entire request. If the HTTP layer of the IPP server completes processing the HTTP headers successfully, it MAY send an intermediate response, such as "100 Continue", with no IPP data before sending the IPP response. A client MUST expect such a variety of responses from an IPP server. For further information on HTTP/1.1, consult the HTTP documents [RFC2616].

An HTTP server MUST support chunking for IPP requests, and an IPP client MUST support chunking for IPP responses according to HTTP/1.1 [RFC2616]. Note: this rule causes a conflict with non-compliant implementations of HTTP/1.1 that don't support chunking for POST methods, and this rule may cause a conflict with non-compliant implementations of HTTP/1.1 that don't support chunking for CGI scripts.

4.1 Printer-uri and job-uri

All Printer and Job objects are identified by a Uniform Resource Identifier (URI) [RFC2396] so that they can be persistently and unambiguously referenced. Since every URL is a specialized form of a URI, even though the more generic term URI is used throughout the rest of this document, its usage is intended to cover the more specific notion of URL as well. 
Some operation elements are encoded twice, once as the request-URI on the HTTP Request-Line and a second time as a REQUIRED operation attribute in the application/ipp entity. These attributes are the target URI for the operation and are called printer-uri and job-uri. Note: The target URI is included twice in an operation referencing the same IPP object, but the two URIs NEED NOT be literally identical. One can be a relative URI and the other can be an absolute URI. HTTP/1.1 allows clients to generate and send a relative URI rather than an absolute URI. A relative URI identifies a resource with the scope of the HTTP server, but does not include scheme, host or port. The following statements characterize how URLs should be used in the mapping of IPP onto HTTP/1.1:

1. Although potentially redundant, a client MUST supply the target of the operation both as an operation attribute and as a URI at the HTTP layer. The rationale for this decision is to maintain a consistent set of rules for mapping application/ipp to possibly many communication layers, even where URLs are not used as the addressing mechanism in the transport layer.

2. Even though these two URLs might not be literally identical (one being relative and the other being absolute), they MUST both reference the same IPP object. However, a Printer NEED NOT verify that the two URLs reference the same IPP object, and NEED NOT take any action if it determines the two URLs to be different.

3. The URI in the HTTP layer is either relative or absolute and is used by the HTTP server to route the HTTP request to the correct resource relative to that HTTP server. The HTTP server need not be aware of the URI within the operation request.

4. Once the HTTP server resource begins to process the HTTP request, it might get the reference to the appropriate IPP Printer object from either the HTTP URI (using to the context of the HTTP server for relative URLS) or from the URI within the operation request; the choice is up to the implementation.

5. HTTP URIs can be relative or absolute, but the target URI in the operation MUST be an absolute URI.

5. IPP URL Scheme

The IPP/1.1 document defines a new scheme 'ipp' as the value of a URL that identifies either an IPP printer object or an IPP job object. The IPP attributes using the 'ipp' scheme are specified below. Because the HTTP layer does not support the 'ipp' scheme, a client MUST map 'ipp' URLs to 'http' URLs, and then follows the HTTP [RFC2616][RFC2617] rules for constructing a Request-Line and HTTP headers. The mapping is simple because the 'ipp' scheme implies all of the same protocol semantics as that of the 'http' scheme 
[RFC2616], except that it represents a print service and the implicit (default) port number that clients use to connect to a server is port 631 .

In the remainder of this section the term 'ipp-URL' means a URL whose scheme is 'ipp' and whose implicit (default) port is 631. The term 'http-URL' means a URL whose scheme is 'http', and the term 'httpsURL' means a URL whose scheme is 'https',

A client and an IPP object (i.e. the server) MUST support the ipp-URL value in the following IPP attributes.

job attributes:

job-uri

job-printer-uri

printer attributes:

printer-uri-supported

operation attributes:

job-uri

printer-uri

Each of the above attributes identifies a printer or job object. The ipp-URL is intended as the value of the attributes in this list, and for no other attributes. All of these attributes have a syntax type of 'uri', but there are attributes with a syntax type of 'uri' that do not use the 'ipp' scheme, e.g. 'job-more-info'.

If a printer registers its URL with a directory service, the printer MUST register an ipp-URL.

User interfaces are beyond the scope of this document. But if software exposes the ipp-URL values of any of the above five attributes to a human user, it is REQUIRED that the human see the ipp-URL as is.

When a client sends a request, it MUST convert a target ipp-URL to a target http-URL for the HTTP layer according to the following rules:

1. change the 'ipp' scheme to 'http'

2. add an explicit port 631 if the URL does not contain an explicit port. Note: port 631 is the IANA assigned Well known Port for the 'ipp' scheme.

The client MUST use the target http-URL in both the HTTP RequestLine and HTTP headers, as specified by HTTP [RFC2616] [RFC2617]. However, the client MUST use the target ipp-URL for the value of the "printer-uri" or "job-uri" operation attribute within the application/ipp body of the request. The server MUST use the ipp-URL 


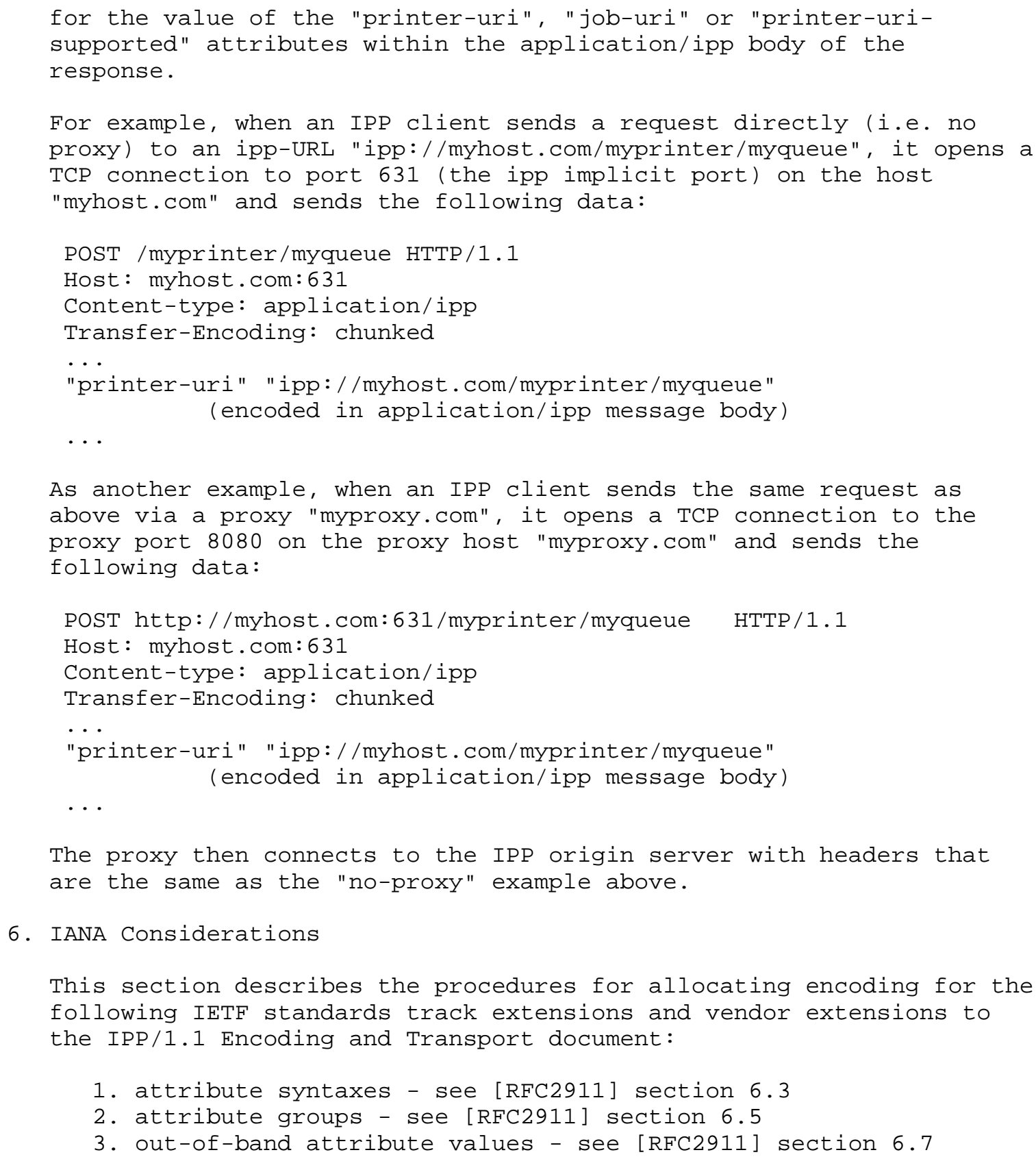


These extensions follow the "type2" registration procedures defined in [RFC2911] section 6. Extensions registered for use with IPP/1.1 are OPTIONAL for client and IPP object conformance to the IPP/1.1 Encoding and Transport document.

These extension procedures are aligned with the guidelines as set forth by the IESG [IANA-CON]. The [RFC2911] Section 11 describes how to propose new registrations for consideration. IANA will reject registration proposals that leave out required information or do not follow the appropriate format described in [RFC2911] Section 11. The IPP/1.1 Encoding and Transport document may also be extended by an appropriate RFC that specifies any of the above extensions.

7. Internationalization Considerations

See the section on "Internationalization Considerations" in the document "Internet Printing Protocol/1.1: Model and Semantics" [RFC2911] for information on internationalization. This document adds no additional issues.

8. Security Considerations

The IPP Model and Semantics document [RFC2911] discusses high level security requirements (Client Authentication, Server Authentication and Operation Privacy). Client Authentication is the mechanism by which the client proves its identity to the server in a secure manner. Server Authentication is the mechanism by which the server proves its identity to the client in a secure manner. Operation Privacy is defined as a mechanism for protecting operations from eavesdropping .

8.1 Security Conformance Requirements

This section defines the security requirements for IPP clients and IPP objects.

\subsubsection{Digest Authentication}

IPP clients MUST support:

Digest Authentication [RFC2617].

MD5 and MD5-sess MUST be implemented and supported.

The Message Integrity feature NEED NOT be used. 
IPP Printers SHOULD support:

Digest Authentication [RFC2617].

MD5 and MD5-sess MUST be implemented and supported.

The Message Integrity feature NEED NOT be used.

The reasons that IPP Printers SHOULD (rather than MUST) support Digest Authentication are:

1. While client Authentication is important, there is a certain class of printer devices where it does not make sense. Specifically, a low-end device with limited ROM space and low paper throughput may not need Client Authentication. This class of device typically requires firmware designers to make trade-offs between protocols and functionality to arrive at the lowest-cost solution possible. Factored into the designer's decisions is not just the size of the code, but also the testing, maintenance, usefulness, and time-tomarket impact for each feature delivered to the customer. Forcing such low-end devices to provide security in order to claim IPP/1.1 conformance would not make business sense and could potentially stall the adoption of the standard.

2. Print devices that have high-volume throughput and have available ROM space have a compelling argument to provide support for Client Authentication that safeguards the device from unauthorized access. These devices are prone to a high loss of consumables and paper if unauthorized access should occur.

\subsubsection{Transport Layer Security (TLS)}

IPP Printers SHOULD support Transport Layer Security (TLS) [RFC2246]

for Server Authentication and Operation Privacy. IPP Printers MAY also support TLS for Client Authentication. If an IPP Printer supports TLS, it MUST support the TLS_DHE_DSS_WITH_3DES_EDE_CBC_SHA cipher suite as mandated by RFC 2246 [RFC2246]. All other cipher suites are OPTIONAL. An IPP Printer MAY support Basic Authentication (described in HTTP/1.1 [RFC2617]) for Client Authentication if the channel is secure. TLS with the above mandated cipher suite can provide such a secure channel.

If a IPP client supports TLS, it MUST support the TLS_DHE_DSS_WITH_3DES_EDE_CBC_SHA cipher suite as mandated by RFC 2246 [RFC2246]. All other cipher suites are OPTIONAL. 
The IPP Model and Semantics document defines two printer attributes ("uri-authentication-supported" and "uri-security-supported") that the client can use to discover the security policy of a printer. That document also outlines IPP-specific security considerations and should be the primary reference for security implications with regard to the IPP protocol itself. For backward compatibility with IPP version 1.0, IPP clients and printers may also support SSL3 [ssl]. This is in addition to the security required in this document.

8.2 Using IPP with TLS

IPP/1.1 uses the "Upgrading to TLS Within HTTP/1.1" mechanism [RFC2817]. An initial IPP request never uses TLS. The client requests a secure TLS connection by using the HTTP "Upgrade" header, while the server agrees in the HTTP response. The switch to TLS occurs either because the server grants the client's request to upgrade to TLS, or a server asks to switch to TLS in its response. Secure communication begins with a server's response to switch to TLS.

9. Interoperability with IPP/1.0 Implementations

It is beyond the scope of this specification to mandate conformance with previous versions. IPP/1.1 was deliberately designed, however, to make supporting previous versions easy. It is worth noting that, at the time of composing this specification (1999), we would expect IPP/1.1 Printer implementations to:

understand any valid request in the format of IPP/1.0, or 1.1;

respond appropriately with a response containing the same "version-number" parameter value used by the client in the request.

And we would expect IPP/1.1 clients to:

understand any valid response in the format of IPP/1.0, or 1.1 .

9.1 The "version-number" Parameter

The following are rules regarding the "version-number" parameter (see section 3.3$)$ :

1. Clients MUST send requests containing a "version-number" parameter with a '1.1' value and SHOULD try supplying alternate version numbers if they receive a 'server-error-version-notsupported' error return in a response. 
2. IPP objects MUST accept requests containing a "version-number" parameter with a '1.1' value (or reject the request for reasons other than 'server-error-version-not-supported').

3. It is recommended that IPP objects accept any request with the major version ' 1 ' (or reject the request for reasons other than ' server-error-version-not-supported'). See [RFC2911]

"versions" sub-section.

4. In any case, security MUST NOT be compromised when a client supplies a lower "version-number" parameter in a request. For example, if an IPP/1.1 conforming Printer object accepts version ' 1.0 ' requests and is configured to enforce Digest Authentication, it MUST do the same for a version '1.0' request.

\subsection{Security and URL Schemes}

The following are rules regarding security, the "version-number" parameter, and the URL scheme supplied in target attributes and responses:

1. When a client supplies a request, the "printer-uri" or "joburi" target operation attribute MUST have the same scheme as that indicated in one of the values of the "printer-urisupported" Printer attribute.

2. When the server returns the "job-printer-uri" or "job-uri" Job Description attributes, it SHOULD return the same scheme ('ipp', 'https', 'http', etc.) that the client supplied in the "printer-uri" or "job-uri" target operation attributes in the Get-Job-Attributes or Get-Jobs request, rather than the scheme used when the job was created. However, when a client requests job attributes using the Get-Job-Attributes or Get-Jobs operations, the jobs and job attributes that the server returns depends on: (1) the security in effect when the job was created, (2) the security in effect in the query request, and (3) the security policy in force.

3. It is recommended that if a server registers a non-secure ippURL with a directory service (see [RFC2911] "Generic Directory Schema" Appendix), then it also register an http-URL for interoperability with IPP/1.0 clients (see section 9).

4. In any case, security MUST NOT be compromised when a client supplies an 'http' or other non-secure URL scheme in the target "printer-uri" and "job-uri" operation attributes in a request. 
10. References

[dpa] ISO/IEC 10175 Document Printing Application (DPA), June 1996 .

[iana] IANA Registry of Coded Character Sets: ftp://ftp.isi.edu/in-notes/iana/assignments/charactersets.

[IANA-CON] Narten, T. and H. Alvestrand, "Guidelines for Writing an IANA Considerations section in RFCs", BCP 26, RFC 2434, October 1998 .

[ipp-iig] Hastings, Tom, et al., "Internet Printing Protocol/1.1: Implementer's Guide", Work in Progress.

[RFC822] Crocker, D., "Standard for the Format of ARPA Internet Text Messages", STD 11, RFC 822, August 1982.

[RFC1123] Braden, S., "Requirements for Internet Hosts - Application and Support", STD 3, RFC 1123, October, 1989.

[RFC1179] MCLaughlin, L. III, (editor), "Line Printer Daemon Protocol", RFC 1179, August 1990.

[RFC2223] Postel, J. and J. Reynolds, "Instructions to RFC Authors", RFC 2223, October 1997.

[RFC1738] Berners-Lee, T., Masinter, L. and M. McCahill, "Uniform Resource Locators (URL)", RFC 1738, December 1994.

[RFC1759] Smith, R., Wright, F., Hastings, T., Zilles, S. and J. Gyllenskog, "Printer MIB", RFC 1759, March 1995.

[RFC1766] Alvestrand, H., "Tags for the Identification of Languages", RFC 1766, March 1995.

[RFC1808] Fielding, R., "Relative Uniform Resource Locators", RFC 1808, June 1995.

[RFC1903] Case, J., McCloghrie, K., Rose, M. and S. Waldbusser, "Textual Conventions for Version 2 of the Simple Network Management Protocol (SNMPv2)", RFC 1903, January 1996.

[RFC2046] Freed, N. and N. Borenstein, "Multipurpose Internet Mail Extensions (MIME) Part Two: Media Types", RFC 2046, November 1996 . 
[RFC2048] Freed, N., Klensin, J. and J. Postel, "Multipurpose Internet Mail Extension (MIME) Part Four: Registration Procedures", BCP 13, RFC 2048, November 1996.

[RFC2119] Bradner, S., "Key words for use in RFCs to Indicate Requirement Levels", BCP 14, RFC 2119, March 1997.

[RFC2184] Freed, N. and K. Moore, "MIME Parameter Value and Encoded Word Extensions: Character Sets, Languages, and Continuations", RFC 2184, August 1997.

[RFC2234] Crocker, D. and P. Overall, "Augmented BNF for Syntax Specifications: ABNF", RFC 2234, November 1997.

[RFC2246] Dierks, T. and C. Allen, "The TLS Protocol", RFC 2246. January 1999 .

[RFC2396] Berners-Lee, T., Fielding, R. and L. Masinter, "Uniform Resource Identifiers (URI) : Generic Syntax", RFC 2396, August 1998 .

[RFC2565] Herriot, R., Butler, S., Moore, P. and R. Turner, "Internet Printing Protocol/1.0: Encoding and Transport", RFC 2565, April 1999.

[RFC2566] deBry, R., Hastings, T., Herriot, R., Isaacson, S. and P. Powell, "Internet Printing Protocol/1.0: Model and Semantics", RFC 2566, April 1999.

[RFC2567] Wright, D., "Design Goals for an Internet Printing Protocol", RFC2567, April 1999.

[RFC2568] Zilles, S., "Rationale for the Structure and Model and Protocol for the Internet Printing Protocol", RFC 2568, April 1999.

[RFC2569] Herriot, R., Hastings, T., Jacobs, N. and J. Martin, "Mapping between LPD and IPP Protocols", RFC 2569, April 1999 .

[RFC2616] Fielding, R., Gettys, J., Mogul, J., Frystyk, H., Masinter, L., Leach, P. and T. Berners-Lee, "Hypertext Transfer Protocol - HTTP/1.1", RFC 2616, June 1999.

[RFC2617] Franks, J., Hallam-Baker, P., Hostetler, J., Lawrence, S., Leach, P., Luotonen, A. and L. Stewart, "HTTP Authentication: Basic and Digest Access Authentication", RFC 2617, June 1999. 
[RFC2817] Khare, R. and S. Lawrence, "Upgrading to TLS Within HTTP/1.1", RFC 2817, May 2000 .

[RFC2910] Herriot, R., Butler, S., Moore, P., Turner, R. and J. Wenn, "Internet Printing Protocol/1.1: Encoding and Transport", RFC 2910, September 2000 .

[RFC2911] Hastings, T., Herriot, R., deBry, R., Isaacson, S. and P. Powell, "Internet Printing Protocol/1.1: Model and Semantics", RFC 2911, September 2000.

$[\mathrm{SSL}]$ Netscape, The SSL Protocol, Version 3, (Text version 3.02), November 1996.

11. Authors' Addresses

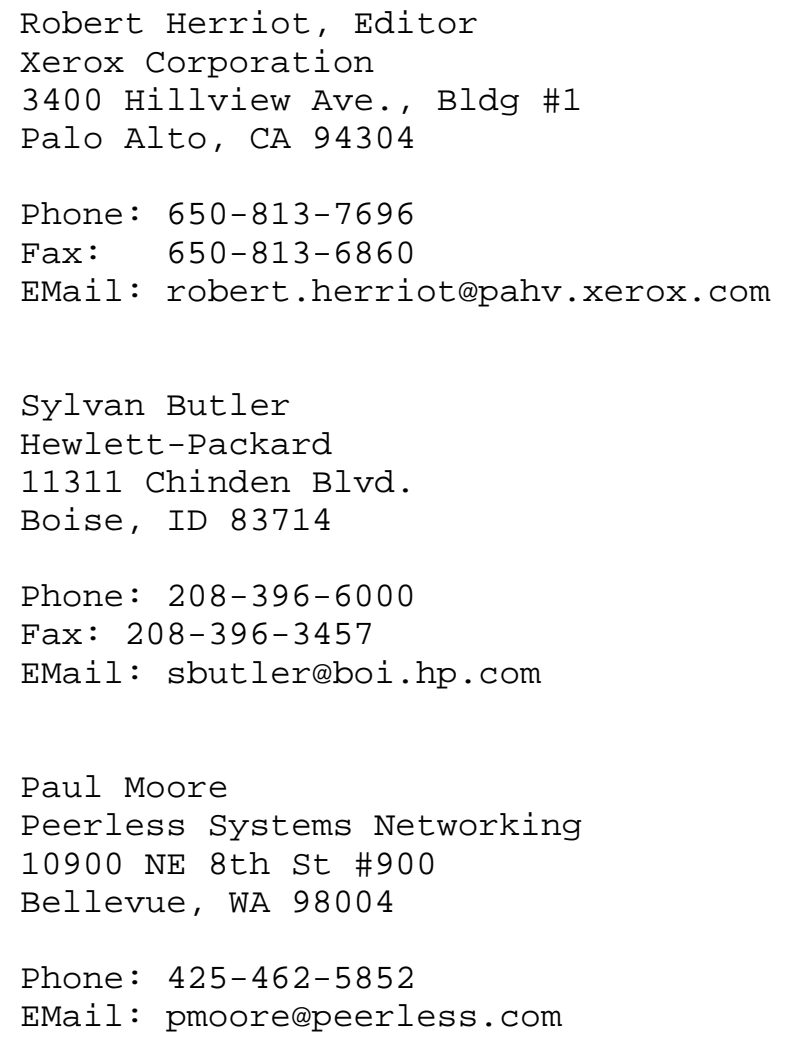




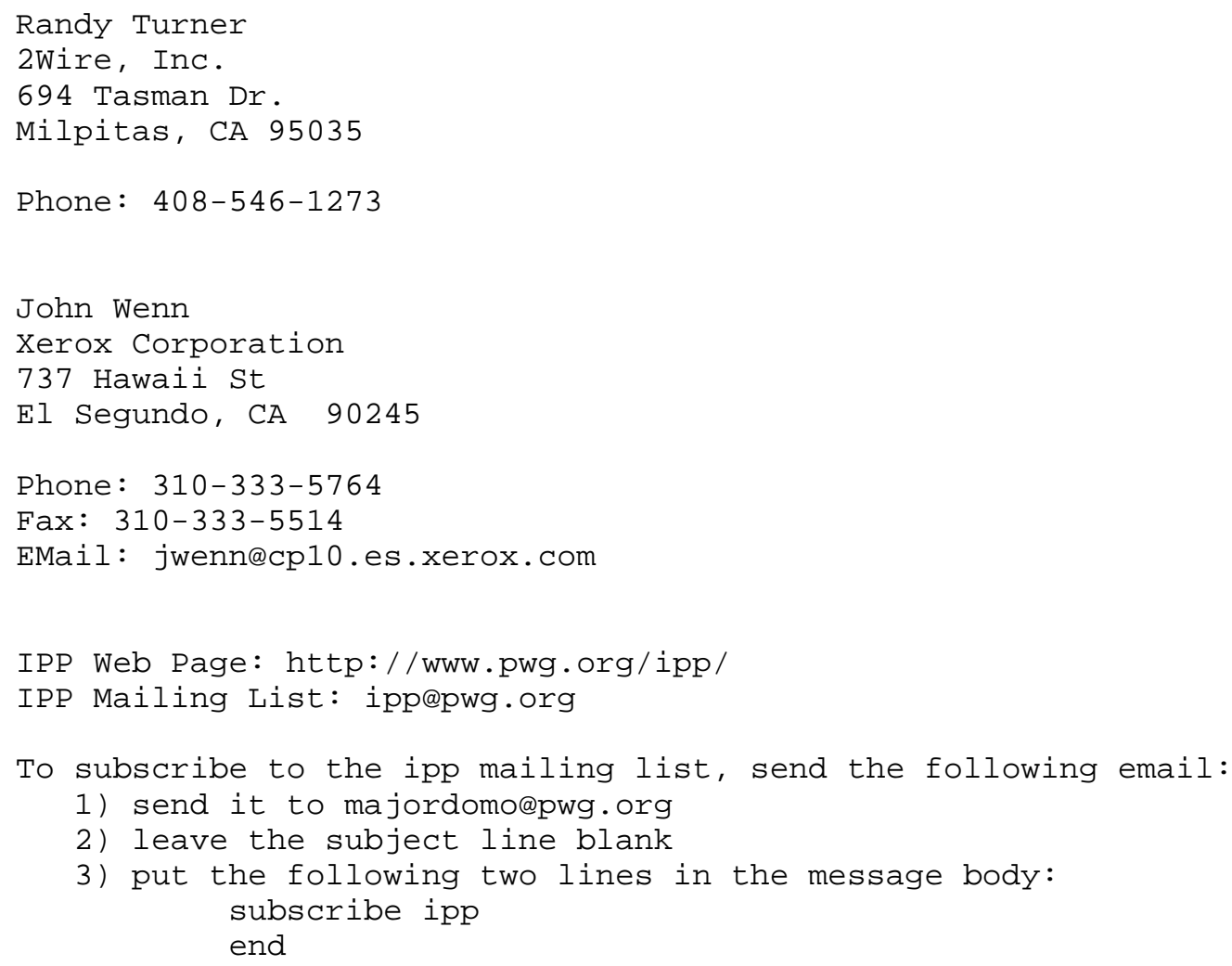


12. Other Participants:

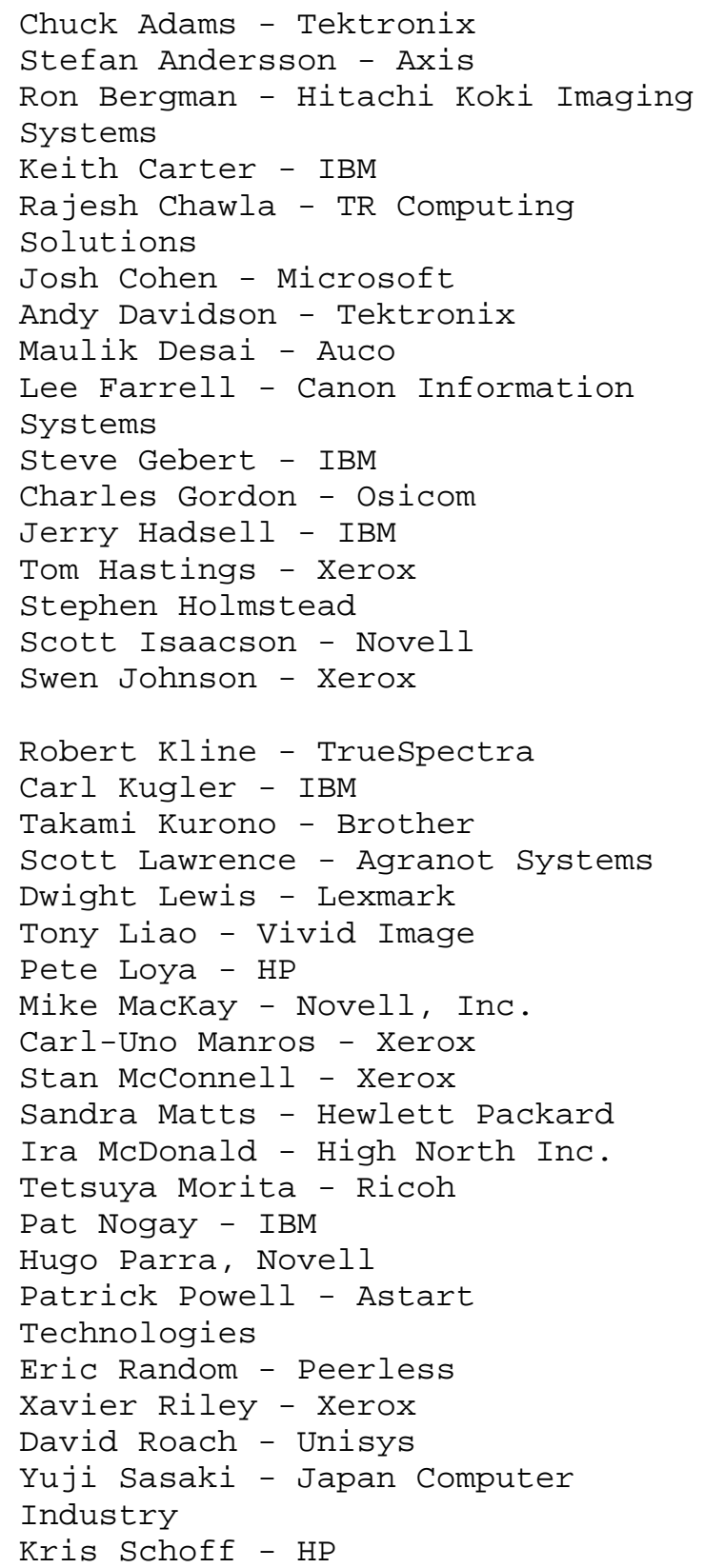




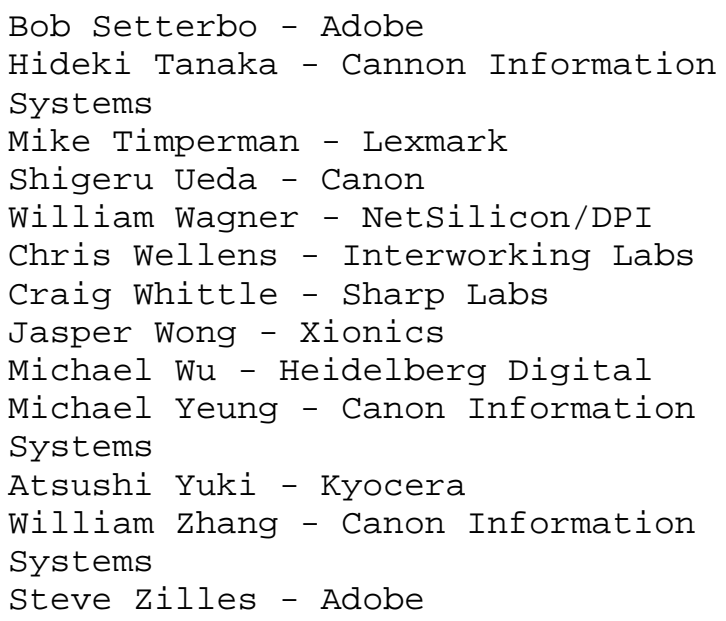

Gail Songer - Peerless

Devon Taylor - Novell, Inc.

Atsushi Uchino - Epson

Bob Von Andel - Allegro Software

Jim Walker - DAZEL

Trevor Wells - Hewlett Packard

Rob Whittle - Novell, Inc.

Don Wright - Lexmark

Rick Yardumian - Xerox

Lloyd Young - Lexmark

Peter Zehler - Xerox

Frank Zhao - Panasonic

Rob Zirnstein - Canon Information systems 


\section{Appendix A: Protocol Examples}

\subsection{Print-Job Request}

The following is an example of a Print-Job request with job-name, copies, and sides specified. The "ipp-attribute-fidelity" attribute is set to 'true' so that the print request will fail if the "copies" or the "sides" attribute are not supported or their values are not supported.

\begin{tabular}{|c|c|c|}
\hline Octets & Symbolic Value & Protocol field \\
\hline $0 \times 0101$ & 1.1 & version-number \\
\hline $0 \times 0002$ & Print-Job & operation-id \\
\hline $0 \times 00000001$ & 1 & request-id \\
\hline $0 \times 01$ & start operation-attributes & operation-attributes-tag \\
\hline $0 \times 47$ & charset type & value-tag \\
\hline $0 \times 0012$ & & name-length \\
\hline $\begin{array}{l}\text { attributes- } \\
\text { charset }\end{array}$ & attributes-charset & name \\
\hline $0 \times 0008$ & & value-length \\
\hline us-ascii & US-ASCII & value \\
\hline $0 \times 48$ & natural-language type & value-tag \\
\hline $\begin{array}{l}\text { 0x001B } \\
\text { attributes- }\end{array}$ & & $\begin{array}{l}\text { name-length } \\
\text { name }\end{array}$ \\
\hline $\begin{array}{l}\text { natural- } \\
\text { language }\end{array}$ & attributes-natural-language & \\
\hline $0 \times 0005$ & & value-length \\
\hline en-us & en-US & value \\
\hline $0 \times 45$ & uri type & value-tag \\
\hline $0 \times 000 B$ & & name-length \\
\hline $\begin{array}{l}\text { printer-uri } \\
0 \times 0015\end{array}$ & printer-uri & $\begin{array}{l}\text { name } \\
\text { value-length }\end{array}$ \\
\hline $\begin{array}{c}\text { ipp: //forest/ } \\
\text { pinetree }\end{array}$ & printer pinetree & value \\
\hline $0 \times 42$ & nameWithoutLanguage type & value-tag \\
\hline $0 \times 0008$ & & name-length \\
\hline job-name & job-name & name \\
\hline $0 \times 0006$ & & value-length \\
\hline foobar & foobar & value \\
\hline $0 \times 22$ & boolean type & value-tag \\
\hline $0 \times 0016$ & & name-length \\
\hline $\begin{array}{l}\text { ipp-attribute- } \\
\text { fidelity }\end{array}$ & ipp-attribute-fidelity & name \\
\hline $0 \times 0001$ & & value-length \\
\hline $0 \times 01$ & true & value \\
\hline
\end{tabular}




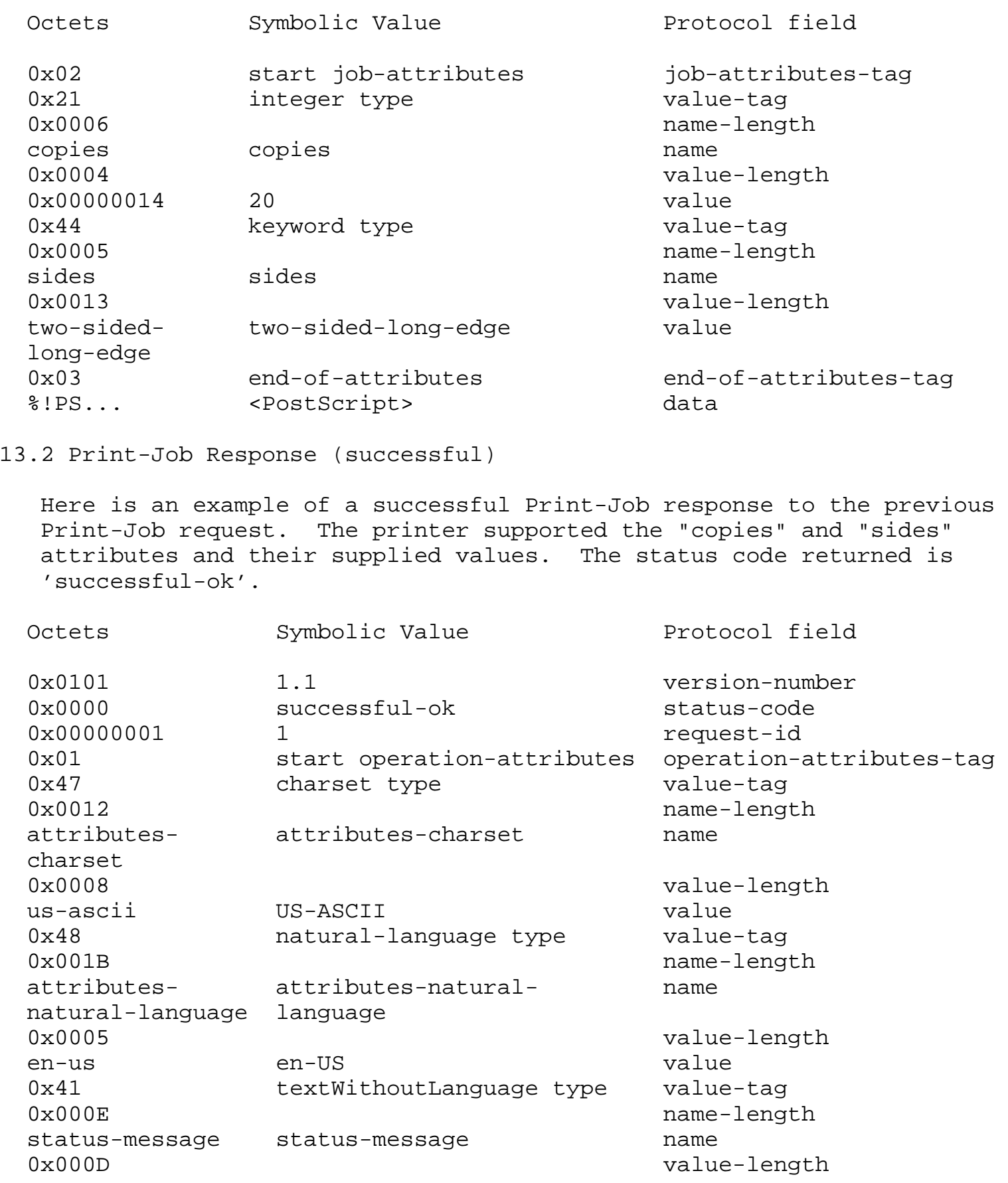




\begin{tabular}{|c|c|c|}
\hline Octets & Symbolic Value & Protocol field \\
\hline successful-ok & successful-ok & value \\
\hline $0 \times 02$ & start job-attributes & job-attributes-tag \\
\hline $0 \times 21$ & integer & value-tag \\
\hline $0 \times 0006$ & & name-length \\
\hline job-id & job-id & name \\
\hline $0 \times 0004$ & & value-length \\
\hline 147 & 147 & value \\
\hline $0 \times 45$ & uri type & value-tag \\
\hline $0 \times 0007$ & & name-length \\
\hline job-uri & job-uri & name \\
\hline $0 \times 0019$ & & value-length \\
\hline $\begin{array}{l}\text { ipp://forest/ } \\
\text { pinetree/123 }\end{array}$ & job 123 on pinetree & value \\
\hline $0 \times 23$ & enum type & value-tag \\
\hline $0 \times 0009$ & & name-length \\
\hline job-state & job-state & name \\
\hline $0 \times 0004$ & & value-length \\
\hline $0 \times 0003$ & pending & value \\
\hline $0 \times 03$ & end-of-attributes & end-of-attributes-tag \\
\hline \multicolumn{3}{|c|}{ 3.3 Print-Job Response (failure) } \\
\hline \multicolumn{3}{|c|}{$\begin{array}{l}\text { Here is an example of an unsuccessful Print-Job response to the } \\
\text { previous Print-Job request. It fails because, in this case, the } \\
\text { printer does not support the "sides" attribute and because the value } \\
\text { '20' for the "copies" attribute is not supported. Therefore, no job } \\
\text { is created, and neither a "job-id" nor a "job-uri" operation } \\
\text { attribute is returned. The error code returned is 'client-error- } \\
\text { attributes-or-values-not-supported' (0x040B). }\end{array}$} \\
\hline $0 \times 0101$ & 1.1 & version-number \\
\hline $0 \times 040 B$ & $\begin{array}{l}\text { client-error-attributes-or- } \\
\text { values-not-supported }\end{array}$ & status-code \\
\hline $0 \times 00000001$ & 1 & request-id \\
\hline $0 \times 01$ & start operation-attributes & operation-attributes tag \\
\hline $0 \times 47$ & charset type & value-tag \\
\hline $0 \times 0012$ & & name-length \\
\hline $\begin{array}{l}\text { attributes- } \\
\text { charset }\end{array}$ & attributes-charset & name \\
\hline $0 \times 0008$ & & value-length \\
\hline us-ascii & $\mathrm{US}-\mathrm{ASCII}$ & value \\
\hline
\end{tabular}




\begin{tabular}{|c|c|c|}
\hline Octets & Symbolic Value & Protocol field \\
\hline $0 \times 48$ & natural-language type & value-tag \\
\hline $0 \times 001 B$ & & name-length \\
\hline $\begin{array}{l}\text { attributes- } \\
\text { natural- }\end{array}$ & attributes-natural-language & name \\
\hline language & & \\
\hline $0 \times 0005$ & & value-length \\
\hline en-us & en-US & value \\
\hline $0 \times 41$ & textWithoutLanguage type & value-tag \\
\hline $0 \times 000 \mathrm{E}$ & & name-length \\
\hline $\begin{array}{l}\text { status- } \\
\text { message }\end{array}$ & status-message & name \\
\hline $0 \times 002 \mathrm{~F}$ & & value-length \\
\hline client-error- & & value \\
\hline attributes- & values-not-supported & \\
\hline $\begin{array}{l}\text { or-values- } \\
\text { not-supported }\end{array}$ & client-error-attributes-or- & \\
\hline $0 \times 05$ & start unsupported-attributes & unsupported-attributes tag \\
\hline $0 \times 21$ & integer type & value-tag \\
\hline $0 \times 0006$ & & name-length \\
\hline copies & copies & name \\
\hline $0 \times 0004$ & & value-length \\
\hline $0 \times 00000014$ & 20 & value \\
\hline $0 \times 10$ & unsupported (type) & value-tag \\
\hline $0 \times 0005$ & & name-length \\
\hline sides & sides & name \\
\hline $0 \times 0000$ & & value-length \\
\hline $0 \times 03$ & end-of-attributes & end-of-attributes-tag \\
\hline 3. 4 Print-Job $\mathrm{R}$ & Response (success with attribu & tes ignored) \\
\hline $\begin{array}{l}\text { Here is an ex } \\
\text { request like } \\
\text { 'ipp-attribut } \\
\text { though, in th } \\
\text { attribute nor } \\
\text { job is create } \\
\text { attribute are } \\
\text { in an Unsuppo } \\
\text { 'successful-c }\end{array}$ & $\begin{array}{l}\text { xample of a successful Print-J } \\
\text { the previous Print-Job reques } \\
\text { te-fidelity' is false. The pri } \\
\text { his case, the printer supports } \\
\text { r the value '20' for the "copi } \\
\text { ed, and both a "job-id" and a } \\
\text { e returned. The unsupported at } \\
\text { orted Attributes Group. The er } \\
\text { ok-ignored-or-substituted-attr }\end{array}$ & $\begin{array}{l}\text { ob response to a Print-Job } \\
\text { t, except that the value of } \\
\text { nt request succeeds, even } \\
\text { neither the "sides" } \\
\text { es" attribute. Therefore, a } \\
\text { "job-uri" operation } \\
\text { tributes are also returned } \\
\text { ror code returned is } \\
\text { ibutes' (0x0001). }\end{array}$ \\
\hline Octets & Symbolic Value & Protocol field \\
\hline $0 \times 0101$ & 1.1 & version-number \\
\hline $0 \times 0001$ & successful-ok-ignored-or- & status-code \\
\hline
\end{tabular}




\begin{tabular}{|c|c|c|}
\hline Octets & Symbolic Value & Protocol field \\
\hline & substituted-attributes & \\
\hline $0 \times 00000001$ & 1 & request-id \\
\hline $0 \times 01$ & start operation-attributes & operation-attributes-tag \\
\hline $0 \times 47$ & charset type & value-tag \\
\hline $0 \times 0012$ & & name-length \\
\hline $\begin{array}{l}\text { attributes- } \\
\text { charset }\end{array}$ & attributes-charset & name \\
\hline $0 \times 0008$ & & value-length \\
\hline us-ascii & US-ASCII & value \\
\hline $0 \times 48$ & natural-language type & value-tag \\
\hline $0 \times 001 B$ & & name-length \\
\hline $\begin{array}{l}\text { attributes- } \\
\text { natural-language }\end{array}$ & $\begin{array}{l}\text { attributes-natural- } \\
\text { language }\end{array}$ & name \\
\hline $0 \times 0005$ & & value-length \\
\hline en-us & en-US & value \\
\hline $\begin{array}{l}0 \times 41 \\
0 \times 000 \mathrm{E}\end{array}$ & textWithoutLanguage type & $\begin{array}{l}\text { value-tag } \\
\text { name-length }\end{array}$ \\
\hline $\begin{array}{l}\text { status-message } \\
0 \times 002 \mathrm{~F}\end{array}$ & status-message & $\begin{array}{l}\text { name } \\
\text { value-length }\end{array}$ \\
\hline $\begin{array}{l}\text { successful-ok- } \\
\text { ignored-or- } \\
\text { substituted- } \\
\text { attributes }\end{array}$ & $\begin{array}{l}\text { successful-ok-ignored-or- } \\
\text { substituted-attributes }\end{array}$ & value \\
\hline $0 \times 05$ & $\begin{array}{l}\text { start unsupported- } \\
\text { attributes }\end{array}$ & $\begin{array}{l}\text { unsupported-attributes } \\
\text { tag }\end{array}$ \\
\hline $0 \times 21$ & integer type & value-tag \\
\hline $0 \times 0006$ & & name-length \\
\hline copies & copies & name \\
\hline $0 \times 0004$ & & value-length \\
\hline $0 \times 00000014$ & 20 & value \\
\hline $0 \times 10$ & unsupported (type) & value-tag \\
\hline $0 \times 0005$ & & name-length \\
\hline sides & sides & name \\
\hline $0 \times 0000$ & & value-length \\
\hline $0 \times 02$ & start job-attributes & job-attributes-tag \\
\hline $0 \times 21$ & integer & value-tag \\
\hline $0 \times 0006$ & & name-length \\
\hline job-id & job-id & name \\
\hline $0 \times 0004$ & & value-length \\
\hline 147 & 147 & value \\
\hline $0 \times 45$ & uri type & value-tag \\
\hline $0 \times 0007$ & & name-length \\
\hline job-uri & job-uri & name \\
\hline $0 \times 0019$ & & value-length \\
\hline ipp: / / forest / & job 123 on pinetree & value \\
\hline
\end{tabular}




$\begin{array}{lll}\text { Octets } & \text { Symbolic Value } & \text { Protocol field } \\ 0 \times 23 & \text { enum type } & \text { value-tag } \\ \text { 0x0009 } & \text { name-length } \\ \text { job-state } & \text { job-state } & \text { name } \\ 0 \times 0004 & \text { value-length } \\ 0 \times 0003 & \text { pending } & \text { value } \\ 0 \times 03 & \text { end-of-attributes } & \text { end-of-attributes-tag }\end{array}$

\subsection{Print-URI Request}

The following is an example of Print-URI request with copies and job-name parameters:

\begin{tabular}{|c|c|c|}
\hline Octets & Symbolic Value & Protocol field \\
\hline $0 \times 0101$ & 1.1 & version-number \\
\hline $0 \times 0003$ & Print-URI & operation-id \\
\hline $0 \times 00000001$ & 1 & request-id \\
\hline $0 \times 01$ & start operation-attributes & operation-attributes-tag \\
\hline $0 \times 47$ & charset type & value-tag \\
\hline $0 \times 0012$ & & name-length \\
\hline $\begin{array}{l}\text { attributes- } \\
\text { charset }\end{array}$ & attributes-charset & name \\
\hline $0 \times 0008$ & & value-length \\
\hline us-ascii & US-ASCII & value \\
\hline $0 \times 48$ & natural-language type & value-tag \\
\hline $0 \times 001 B$ & & name-length \\
\hline $\begin{array}{l}\text { attributes- } \\
\text { natural- } \\
\text { language }\end{array}$ & attributes-natural-language & name \\
\hline $0 \times 0005$ & & value-length \\
\hline en-us & en-US & value \\
\hline $0 \times 45$ & uri type & value-tag \\
\hline $0 \times 000 B$ & & name-length \\
\hline $\begin{array}{l}\text { printer-uri } \\
0 \times 0015\end{array}$ & printer-uri & $\begin{array}{l}\text { name } \\
\text { value-length }\end{array}$ \\
\hline $\begin{array}{c}\text { ipp: //forest/ } \\
\text { pinetree }\end{array}$ & printer pinetree & value \\
\hline $0 \times 45$ & uri type & value-tag \\
\hline $0 \times 000 \mathrm{C}$ & & name-length \\
\hline document-uri & document-uri & name \\
\hline $0 \times 0011$ & & value-length \\
\hline ftp: / / foo.com & ftp://foo.com/foo & value \\
\hline
\end{tabular}




\begin{tabular}{|c|c|c|}
\hline Octets & Symbolic Value & Protocol field \\
\hline \multicolumn{3}{|l|}{ / foo } \\
\hline $0 \times 42$ & nameWithoutLanguage type & value-tag \\
\hline $0 \times 0008$ & & name-length \\
\hline job-name & job-name & name \\
\hline $0 \times 0006$ & & value-length \\
\hline foobar & foobar & value \\
\hline $0 \times 02$ & start job-attributes & job-attributes-tag \\
\hline $0 \times 21$ & integer type & value-tag \\
\hline $0 \times 0006$ & & name-length \\
\hline copies & copies & name \\
\hline $0 \times 0004$ & & value-length \\
\hline $0 \times 00000001$ & 1 & value \\
\hline $0 \times 03$ & end-of-attributes & end-of-attributes-tag \\
\hline
\end{tabular}

13. 6 Create-Job Request

The following is an example of Create-Job request with no parameters and no attributes:

\begin{tabular}{|c|c|c|}
\hline Octets & Symbolic Value & Protocol field \\
\hline $0 \times 0101$ & 1.1 & version-number \\
\hline $0 \times 0005$ & Create-Job & operation-id \\
\hline $0 \times 00000001$ & 1 & request-id \\
\hline $0 \times 01$ & start operation-attributes & operation-attributes-tag \\
\hline $0 \times 47$ & charset type & value-tag \\
\hline $0 \times 0012$ & & name-length \\
\hline $\begin{array}{l}\text { attributes- } \\
\text { charset }\end{array}$ & attributes-charset & name \\
\hline $0 \times 0008$ & & value-length \\
\hline us-ascii & $\mathrm{US}-\mathrm{ASCII}$ & value \\
\hline $0 \times 48$ & natural-language type & value-tag \\
\hline $0 \times 001 B$ & & name-length \\
\hline $\begin{array}{l}\text { attributes- } \\
\text { natural- } \\
\text { language }\end{array}$ & attributes-natural-language & name \\
\hline $0 \times 0005$ & & value-length \\
\hline en-us & en-US & value \\
\hline $\begin{array}{l}0 \times 45 \\
0 \times 000 B\end{array}$ & uri type & $\begin{array}{l}\text { value-tag } \\
\text { name-length }\end{array}$ \\
\hline $\begin{array}{l}\text { printer-uri } \\
0 \times 0015\end{array}$ & printer-uri & $\begin{array}{l}\text { name } \\
\text { value-length }\end{array}$ \\
\hline ipp: //forest/ & printer pinetree & value \\
\hline
\end{tabular}




$\begin{array}{lll}\text { Octets } & \text { Symbolic Value } & \text { Protocol field } \\ \begin{array}{lll}\text { inetree } & \text { end-of-attributes } & \text { end-of-attributes-tag }\end{array}\end{array}$

\subsection{Get-Jobs Request}

\begin{tabular}{|c|c|c|}
\hline Octets & Symbolic Value & Protocol field \\
\hline $0 \times 0101$ & 1.1 & version-number \\
\hline $0 \times 000 A$ & Get-Jobs & operation-id \\
\hline $0 \times 00000123$ & $0 \times 123$ & request-id \\
\hline $0 \times 01$ & start operation-attributes & operation-attributes-tag \\
\hline $\begin{array}{l}0 \times 47 \\
0 \times 0012\end{array}$ & charset type & $\begin{array}{l}\text { value-tag } \\
\text { name-length }\end{array}$ \\
\hline $\begin{array}{l}\text { attributes- } \\
\text { charset }\end{array}$ & attributes-charset & name \\
\hline $0 \times 0008$ & & value-length \\
\hline us-ascii & US-ASCII & value \\
\hline $\begin{array}{l}0 \times 48 \\
0 \times 001 B\end{array}$ & natural-language type & $\begin{array}{l}\text { value-tag } \\
\text { name-length }\end{array}$ \\
\hline $\begin{array}{l}\text { attributes- } \\
\text { natural- } \\
\text { language }\end{array}$ & attributes-natural-language & name \\
\hline $0 \times 0005$ & & value-length \\
\hline en-us & en-US & value \\
\hline $\begin{array}{l}0 \times 45 \\
0 \times 000 B\end{array}$ & uri type & $\begin{array}{l}\text { value-tag } \\
\text { name-length }\end{array}$ \\
\hline $\begin{array}{l}\text { printer-uri } \\
0 \times 0015\end{array}$ & printer-uri & $\begin{array}{l}\text { name } \\
\text { value-length }\end{array}$ \\
\hline $\begin{array}{l}\text { ipp: //forest/ } \\
\text { pinetree }\end{array}$ & printer pinetree & value \\
\hline $\begin{array}{l}0 \times 21 \\
0 \times 0005\end{array}$ & integer type & $\begin{array}{l}\text { value-tag } \\
\text { name-length }\end{array}$ \\
\hline $\begin{array}{l}\text { limit } \\
0 \times 0004\end{array}$ & limit & $\begin{array}{l}\text { name } \\
\text { value-length }\end{array}$ \\
\hline $0 \times 00000032$ & 50 & value \\
\hline $\begin{array}{l}0 \times 44 \\
0 \times 0014\end{array}$ & keyword type & $\begin{array}{l}\text { value-tag } \\
\text { name-length }\end{array}$ \\
\hline $\begin{array}{l}\text { requested- } \\
\text { attributes }\end{array}$ & requested-attributes & name \\
\hline $0 \times 0006$ & & value-length \\
\hline
\end{tabular}




$\begin{array}{lll}\text { Octets } & \text { Symbolic Value } & \text { Protocol field } \\ \text { job-id } & \text { job-id } & \\ \text { 0x44 } & \text { keyword type } & \text { value } \\ \text { 0x0000 } & \text { value-tag } \\ \text { 0x0008 } & \text { name-length } \\ \text { job-name } & \text { job-name } & \text { value-length } \\ \text { 0x44 } & \text { keyword type } & \text { value } \\ \text { 0x0000 } & \text { additional value } & \text { value-tag } \\ \text { 0x000F } & \text { name-length } \\ \text { document-format } & \text { document-format } & \text { value-length } \\ \text { 0x03 } & \text { end-of-attributes } & \text { value } \\ & & \text { end-of-attributes-tag }\end{array}$

\subsection{Get-Jobs Response}

The following is an of Get-Jobs response from previous request with 3 jobs. The Printer returns no information about the second job (because of security reasons):

\begin{tabular}{|c|c|c|}
\hline Octets & Symbolic Value & Protocol field \\
\hline $0 \times 0101$ & 1.1 & version-number \\
\hline $0 \times 0000$ & successful-ok & status-code \\
\hline $0 \times 00000123$ & $0 \times 123$ & $\begin{array}{l}\text { request-id (echoed } \\
\text { back) }\end{array}$ \\
\hline $0 \times 01$ & start operation-attributes & operation-attributes-tag \\
\hline $0 \times 47$ & charset type & value-tag \\
\hline $0 \times 0012$ & & name-length \\
\hline $\begin{array}{l}\text { attributes- } \\
\text { charset }\end{array}$ & attributes-charset & name \\
\hline $0 \times 000 A$ & & value-length \\
\hline ISO-8859-1 & ISO-8859-1 & value \\
\hline $0 \times 48$ & natural-language type & value-tag \\
\hline $0 \times 001 B$ & & name-length \\
\hline $\begin{array}{l}\text { attributes- } \\
\text { natural- } \\
\text { language }\end{array}$ & attributes-natural-language & name \\
\hline $0 \times 0005$ & & value-length \\
\hline en-us & en-US & value \\
\hline $0 \times 41$ & textWithoutLanguage type & value-tag \\
\hline $0 \times 000 \mathrm{E}$ & & name-length \\
\hline $\begin{array}{l}\text { status-message } \\
\text { 0x000D }\end{array}$ & status-message & $\begin{array}{l}\text { name } \\
\text { value-length }\end{array}$ \\
\hline successful-ok & successful-ok & value \\
\hline $0 \times 02$ & start job-attributes (1st & job-attributes-tag \\
\hline
\end{tabular}




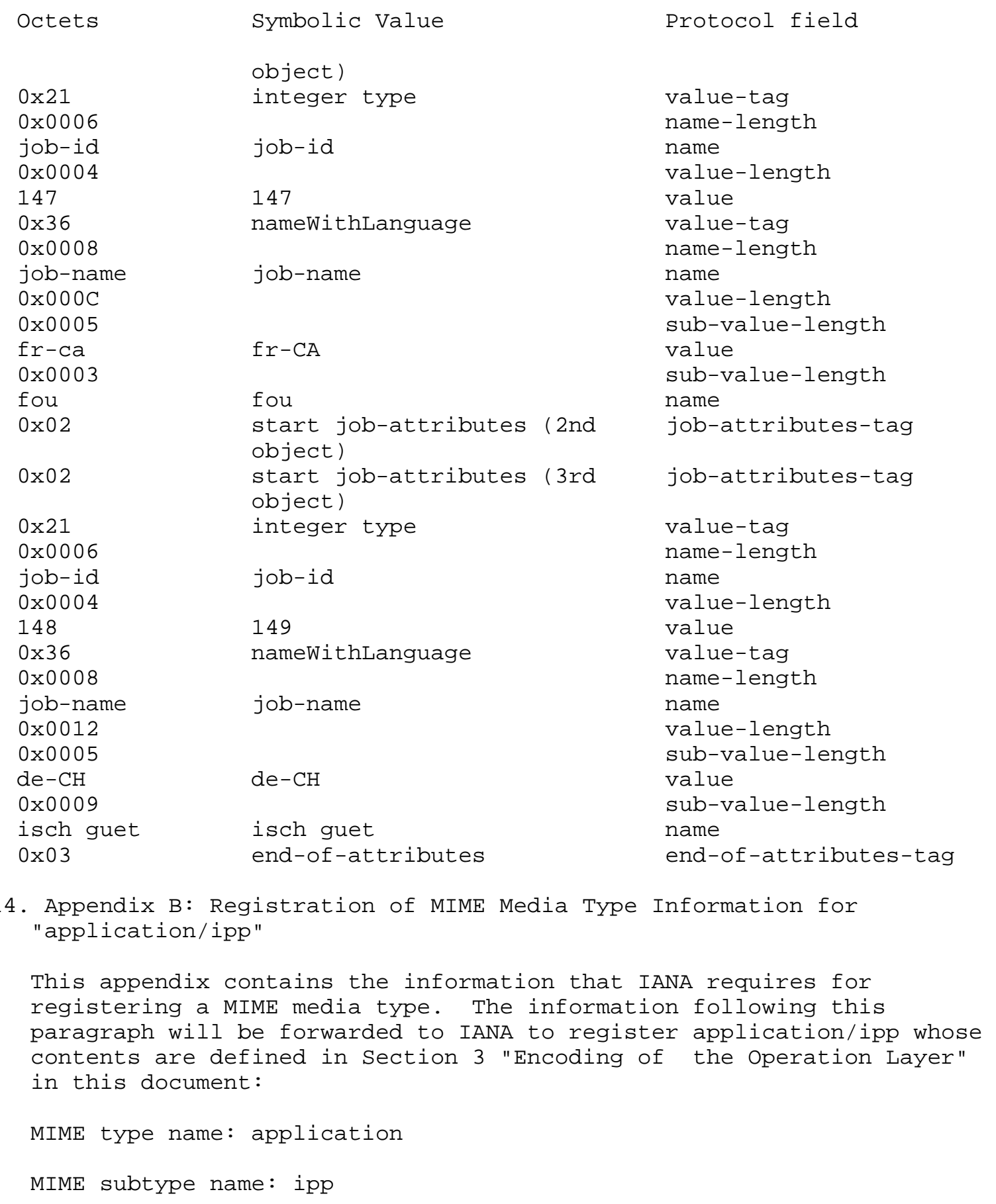


A Content-Type of "application/ipp" indicates an Internet Printing Protocol message body (request or response). Currently there is one version: IPP/1.1, whose syntax is described in section 3 "Encoding of the Operation Layer" of [RFC2910], and whose semantics are described in [RFC2911].

Required parameters: none

Optional parameters: none

Encoding considerations:

IPP/1.1 protocol requests/responses MAY contain long lines and ALWAYS contain binary data (for example attribute value lengths).

Security considerations:

IPP/1.1 protocol requests/responses do not introduce any security risks not already inherent in the underlying transport protocols. Protocol mixed-version interworking rules in [RFC2911] as well as protocol encoding rules in [RFC2910] are complete and unambiguous.

Interoperability considerations:

IPP/1.1 requests (generated by clients) and responses (generated by servers) MUST comply with all conformance requirements imposed by the normative specifications [RFC2911] and [RFC2910]. Protocol encoding rules specified in [RFC2910] are comprehensive, so that interoperability between conforming implementations is guaranteed (although support for specific optional features is not ensured). Both the "charset" and "natural-language" of all IPP/1.1 attribute values which are a LOCALIZED-STRING are explicit within IPP protocol requests/responses (without recourse to any external information in HTTP, SMTP, or other message transport headers).

Published specifications:

[RFC2911] Hastings, T., Herriot, R., deBry, R., Isaacson, S. and P. Powell, "Internet Printing Protocol/1.1: Model and Semantics", RFC 2911, September 2000.

[RFC2910] Herriot, R., Butler, S., Moore, P., Turner, R. and J. Wenn, "Internet Printing Protocol/1.1: Encoding and Transport", RFC 2910, September 2000. 


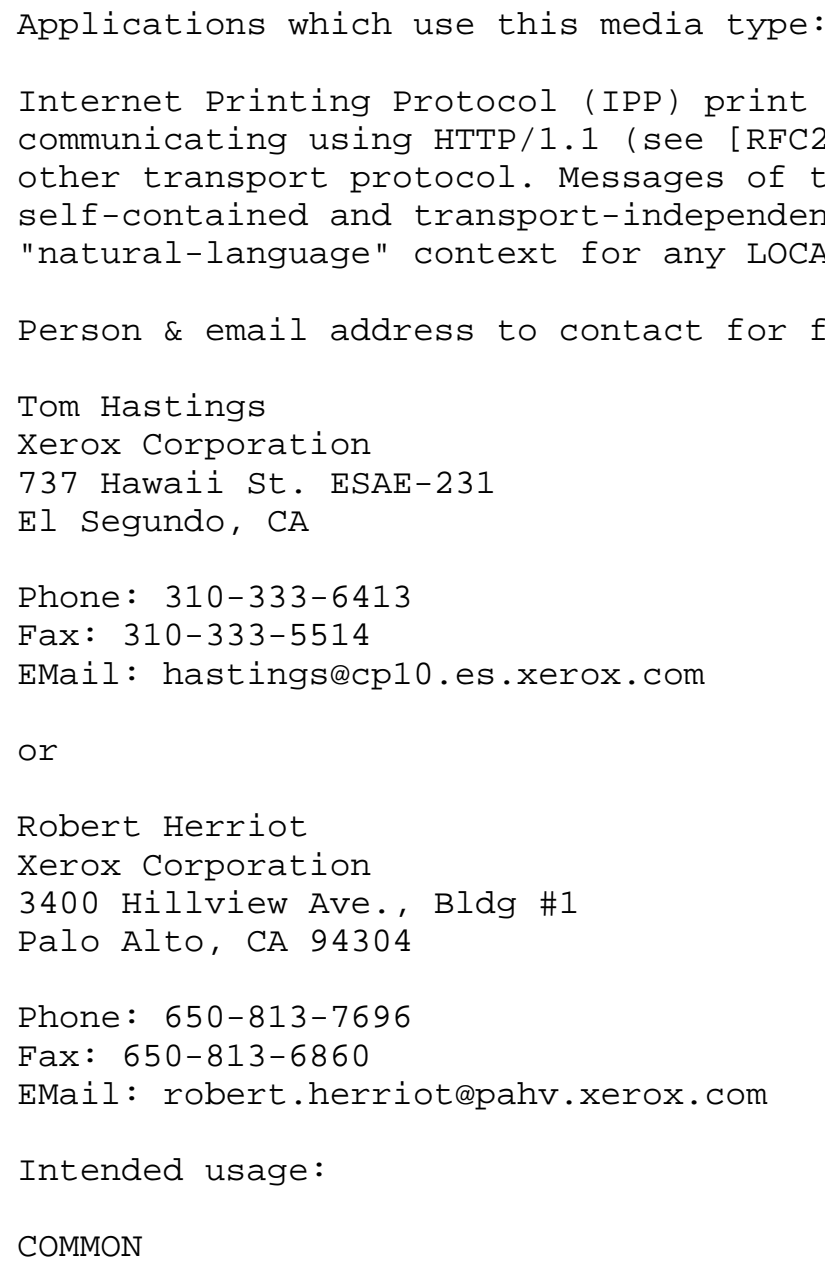

2. Clients MUST support of Digest Authentication, IPP Printers SHOULD support Digest Authentication. See Section 8.1.1

3. TLS is recommended for channel security. In addition, SSL3 may be supported for backward compatibility. See Section 8.1.2 
4. It is recommended that IPP/1.1 objects accept any request with major version number ' ${ }^{\prime}$. See section 9.1 .

5. IPP objects SHOULD return the URL scheme requested for "jobprinter-uri" and "job-uri" Job Attributes, rather than the URL scheme used to create the job. See section 9.2.

6. The IANA and Internationalization sections have been added. The terms "private use" and "experimental" have been changed to "vendor extension". The reserved allocations for attribute group tags, attribute syntax tags, and out-of-band attribute values have been clarified as to which are reserved to future IETF standards track documents and which are reserved to vendor extension. Both kinds of extensions use the type2 registration procedures as defined in [RFC2911].

7. Clarified that future "out-of-band" value definitions may use the value field if additional information is needed. 
Full Copyright statement

Copyright (C) The Internet Society (2000). All Rights Reserved.

This document and translations of it may be copied and furnished to others, and derivative works that comment on or otherwise explain it or assist in its implementation may be prepared, copied, published and distributed, in whole or in part, without restriction of any kind, provided that the above copyright notice and this paragraph are included on all such copies and derivative works. However, this document itself may not be modified in any way, such as by removing the copyright notice or references to the Internet society or other Internet organizations, except as needed for the purpose of developing Internet standards in which case the procedures for copyrights defined in the Internet Standards process must be followed, or as required to translate it into languages other than English.

The limited permissions granted above are perpetual and will not be revoked by the Internet society or its successors or assigns.

This document and the information contained herein is provided on an "AS IS" basis and THE INTERNET SOCIETY AND THE INTERNET ENGINEERING TASK FORCE DISCLAIMS ALL WARRANTIES, EXPRESS OR IMPLIED, INCLUDING BUT NOT LIMITED TO ANY WARRANTY THAT THE USE OF THE INFORMATION HEREIN WILL NOT INFRINGE ANY RIGHTS OR ANY IMPLIED WARRANTIES OF MERCHANTABILITY OR FITNESS FOR A PARTICULAR PURPOSE.

Acknowledgement

Funding for the RFC Editor function is currently provided by the Internet society. 